\title{
Advances in the Universal NMR Database Approach. 2'-Substituted Taxanes as Probes for an Improved Protocol of Diastereomeric Differentiation
}

\author{
Paolo Dambruoso, ${ }^{\mathrm{a}, \mathrm{b}, \dagger}$ Carla Bassarello, ${ }^{\mathrm{b}}$ Giuseppe Bifulco, ${ }^{\mathrm{b}}$ Giovanni Appendino, ${ }^{\mathrm{a}}$ Arturo \\ Battaglia, ${ }^{c}$ Gabriele Fontana, ${ }^{d}$ and Luigi Gomez-Paloma*,b
}

a) DISCAFF, Facoltà di Farmacia, Università del Piemonte Orientale, Largo Donegani, 2 - 28100 - Novara -Italy; b) Dipartimento di Scienze Farmaceutiche, Facoltà di Farmacia, Università di Salerno, Via Ponte don Melillo - 84084 Fisciano (SA) - Italy; c) ISOF - CNR, Area della Ricerca di Bologna, Via P. Gobetti, 101 - 40129 - Bologna - Italy; d) Indena SpA, Viale Ortles 12, 20139 - Milano - Italy.

† Present address: Laboratorio di Chimica Organica, Dipartimento di Chimica, Università di Ferrara, via Borsari 46, 44100-Ferrara-Italy.

gomez@unisa.it

\section{SUPPLEMENTARY MATERIAL}

INDEX

General page 3

${ }^{1} \mathrm{H}$ and ${ }^{13} \mathrm{C}$ c.s. values of the epimeric pair $\mathbf{3 a}, \mathbf{b}$

Table S1

${ }^{1} \mathrm{H}$ and ${ }^{13} \mathrm{C}$ c.s. values of the epimeric pair $\mathbf{4 a} \mathbf{a}, \mathbf{b}$

Table S2

${ }^{1} \mathrm{H}$ and ${ }^{13} \mathrm{C}$ c.s. values of the epimeric pair $\mathbf{5 a}, \mathbf{b}$

Table S3

${ }^{1} \mathrm{H}$ and ${ }^{13} \mathrm{C}$ c.s. values of the epimeric pair $\mathbf{6 a}, \mathbf{b}$

Table S4

${ }^{1} \mathrm{H}$ and ${ }^{13} \mathrm{C}$ c.s. values of the epimeric pair $7 \mathbf{a}, \mathbf{b}$

Table S5

Measured ${ }^{2,3} \mathrm{~J}_{\mathrm{HC}}$, ROESY, TAD and RI values for all 2'-substituted taxanes

Table S6

page 4

page 4

page 5

page 5

page 6

page 7

\section{2'-Ethyltaxanes}

(database elements: epimeric 2'-methyl taxanes)

Side chain ${ }^{13} \mathrm{C}$ c.s. and averaged values for database elements and unknown epimers Original UDB analysis

Table S7

Table S8

Modified UDB analysis

Table S9

page 8

page 8

page 9

\section{2'-Ethyltaxanes}

(database elements: epimeric methyl 2-methylisoserinate)

Side chain ${ }^{13} \mathrm{C}$ c.s. and averaged values for database elements and unknown epimers Modified UDB analysis

Table S10

page 10

Table S11 ............. page 10

\section{2'-Isopropyltaxanes}

Side chain ${ }^{13} \mathrm{C}$ c.s. and averaged values for database elements and unknown epimers Original UDB analysis

Table S12

Table S13 Modified UDB analysis

Table S14

page 11

page 11

page 12

\section{2'-tertButyltaxanes}

(Comparison of the major conformers) 
Side chain ${ }^{13} \mathrm{C}$ c.s. and averaged values for database elements and unknown epimers

Original UDB analysis

Table S15

Table S16

Table S17

page 13

Modified UDB analysis

page 13

page 14

\section{2'-tertButyltaxanes}

(Comparison of the minor conformers)

Side chain ${ }^{13} \mathrm{C}$ c.s. and averaged values for database elements and unknown epimers .......

Original UDB analysis

Modified UDB analysis

Histograms of modified UDB analysis

Table S18

Table S19

Table S20

Figure $\mathrm{S} 1$

page 15

page 15

page 16

\section{2'-tertButyltaxanes}

(Comparison of the major conformer of epimer 1 with the minor conformer of epimer 2)

Side chain ${ }^{13} \mathrm{C}$ c.s. and averaged values for database elements and unknown epimers

Table S21

Table S22

Original UDB analysis

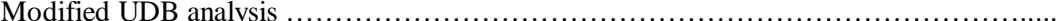

Histograms of modified UDB analysis

Table S23

Figure S2

page 17

page 17

page 18

page 18

\section{2'-tertButyltaxanes}

(Comparison of the minor conformer of epimer 1 with the major conformer of epimer 2)

Side chain ${ }^{13} \mathrm{C}$ c.s. and averaged values for database elements and unknown epimers

Table S24

Table S25

Original UDB analysis

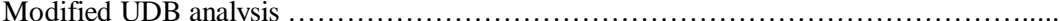

Histograms of modified UDB analysis

Table S26

Figure S3

page 19

page 19

page 20

page 20

\section{2'-Phenyltaxanes}

Side chain ${ }^{13} \mathrm{C}$ c.s. and averaged values for database elements and unknown epimers ....... Original UDB analysis

Table S27

Table S28

Table S29

page 21

page 21

Modified UDB analysis

page 22

\section{2'-Vinyltaxanes}

Side chain ${ }^{13} \mathrm{C}$ c.s. and averaged values for database elements and unknown epimers

Table S30

page 23

Original UDB analysis

Table S31

page 23

Modified UDB analysis

Table S32

page 24 


\section{General}

NMR measurements were performed on a Bruker DRX-600 at $T=300 \mathrm{~K}$. All spectra were acquired in the phase-sensitive mode and the TPPI method was used for quadrature detection in the $\omega_{1}$ dimension.

NMR samples were prepared dissolving each 2'-substituted taxane in $500 \mu \mathrm{L}$ of $\mathrm{CDCl}_{3}$ (Carlo Erba, 99.95\% D) affording the following concentration ranges: (a) 75-120 mM for compounds $\mathbf{2 a}, \mathbf{2 b}, \mathbf{3 a}$, 3b, 4b, 5a, 5b, 7a and 7b; (b) 40-60 mM: 4a and $\mathbf{6 b}$; (c) $32 \mathrm{mM}$ for compound $\mathbf{6 a}$. The spectra were calibrated using the solvent signal as internal standard $\left({ }^{1} \mathrm{H}, \delta=7.26 \mathrm{ppm} ;{ }^{13} \mathrm{C}, \delta=77.0 \mathrm{ppm}\right)$. For compounds belonging to classes (a) and (b), ${ }^{2,3} J_{\mathrm{C}-\mathrm{H}}$ values were obtained from phase-sensitive PFG-PS-HMBC spectra (J. Boyd, N. Soffe, B. John, D. Plant, R. Hurd, J. Magn. Reson. 1992, 98, 660-664; A. L. Davis, J. Keeler, E. D. Laue, D. Moskau, J. Magn. Reson. 1992, 98, 207-216) with a number of scans/ $t_{1}$ ranging from 32 to128, acquiring $4 \mathrm{~K}$ points in $\omega_{2}$, and with $\mathrm{t}_{1 \max }$ values in the range of 2.4-9 ms, while for $6 \mathbf{a}, 256 \mathrm{scans} / \mathrm{t}_{1}$ were acquired with $4 \mathrm{~K}$ points in $\omega_{2}$ and with $\mathrm{t}_{1 \max }$ values in the $5.0 \mathrm{~ms}$ range. The delay for long-range coupling evolution $(\Delta)$ was set at $50 \mathrm{~ms}$. Upon 2D-FT, zero-filling $(4 \mathrm{Kx} 1 \mathrm{~K})$ was carried out in $\omega_{2}$ and $\omega_{1}$. Phase-sensitive PFG-HETLOC spectra (Uhrín, D.; Batta, G.; Hruby, V. J.; Barlow, P. N.; Kövér, K. E. J. Magn. Reson., 1998, 130, 155 and references cited therein) were acquired for all compounds with a number of scans $/ \mathrm{t}_{1}$ ranging from 32 to $128,4 \mathrm{~K}$ points in $\omega_{2}$, a spin lock of $50 \mathrm{~ms}$ and $\mathrm{t}_{1 \max }$ values in the range of $12-50 \mathrm{~ms}$. The data matrices were zero-filled to $4 \mathrm{Kx} 1 \mathrm{~K}$ affording a digital resolution of $1.3 \mathrm{~Hz}$ in $\omega_{2}$.

For the ROESY spectra (Bax, A.; Davis, D. G. J. Magn. Reson., 1985, 63, 207-213) the following acquisition conditions were set: $8-64 \mathrm{scans} / \mathrm{t}_{1}, \mathrm{t}_{1 \max }$ values of $15-50 \mathrm{~ms}$ and a mixing time di $400 \mathrm{~ms}$. The NMR data were processed on a Silicon Graphic Indigo2 workstation using the Bruker XWINNMR software 
$\underline{\text { Table S1: }}{ }^{11} \mathrm{H}$ and ${ }^{13} \mathrm{C}$ c.s. values of the epimeric pair $\mathbf{3 a}, \mathbf{b}$

\begin{tabular}{|c|c|c|c|c|}
\hline & \multicolumn{2}{|c|}{ Minor (3a) } & \multicolumn{2}{|c|}{ Major (3b) } \\
\hline & ${ }^{1} \mathrm{H}$ NMR & ${ }^{13} \mathrm{C}$ NMR & ${ }^{1} \mathrm{H}$ NMR & ${ }^{13} \mathrm{C}$ NMR \\
\hline C1 & I & 79,56 & I & 79,60 \\
\hline $\mathrm{C} 2$ & 5,65 & 75,28 & 5,68 & 75,22 \\
\hline $\mathrm{C} 2(\mathrm{CO})$ & I & 167,47 & I & 167,47 \\
\hline C2 (C(iv)) & I & 129,54 & I & 129,44 \\
\hline C2 (C-orto) & 8,15 & 130,73 & 8,07 & 130,39 \\
\hline C2 (C-meta) & 7,46 & 128,91 & 7,46 & 128,96 \\
\hline C2 (C-para) & 7,57 & 133,78 & 7,60 & 134,08 \\
\hline C3 & 3,82 & 46,89 & 3,82 & 47,00 \\
\hline $\mathrm{C} 4$ & I & 81,20 & 1 & 81,15 \\
\hline C4 (Acetyl-CO) & I & 170,95 & l & 170,77 \\
\hline $\mathrm{C} 4$ (Acetyl-CH3) & 2,53 & 23,11 & 2,49 & 22,92 \\
\hline C5 & 4,91 & 84,61 & 4,91 & 84,70 \\
\hline C6 & 1,88 & 37,53 & 1,88 & 37,47 \\
\hline & 2,51 & I & 2,50 & I \\
\hline C7 & 4,44 & 72,51 & 4,47 & 72,63 \\
\hline $\mathrm{C} 7$ (CH2-TES) & 0,56 & 5,55 & 0,55 & 5,60 \\
\hline C7 (CH3-TES) & 0,91 & 7,05 & 0,90 & 7,05 \\
\hline C8 & I & 58,64 & I & 58,72 \\
\hline C9 & I & 202,05 & I & 202,05 \\
\hline C10 & 6,43 & 75,18 & 6,44 & 75,22 \\
\hline C10 (Acetyl-CO) & I & 169,58 & I & 169,64 \\
\hline $\mathrm{Cl0}$ (Acetyl-CH3) & 2,16 & 21,20 & 2,17 & 21,21 \\
\hline C11 & I & 133,62 & I & 133,60 \\
\hline $\mathrm{C} 12$ & I & 140,59 & I & 140,43 \\
\hline C13 & 6,36 & 72,41 & 6,31 & 72,45 \\
\hline C14 & 2,08 & 36,01 & 2,11 & 35,81 \\
\hline & 2,32 & I & 2,27 & I \\
\hline C15 & I & 43,65 & I & 43,71 \\
\hline C16 & 1,21 & 22,05 & 1,23 & 21,90 \\
\hline C17 & 1,23 & 26,93 & 1,24 & 27,00 \\
\hline C18 & 2,01 & 14,61 & 2,06 & 15,34 \\
\hline C19 & 1,68 & 10,45 & 1,69 & 10,44 \\
\hline C20 & 4,17 & 76,92 & 4,13 & 76,92 \\
\hline & 4,28 & I & 4,30 & I \\
\hline $\mathrm{Cl}^{\prime}$ & I & 176,34 & I & 175,67 \\
\hline $\mathrm{C}^{\prime}$ & I & 81,75 & I & 81,94 \\
\hline $\mathrm{C3}^{\prime}$ & 4,00 & 54,07 & 4,06 & 54,27 \\
\hline $\mathbf{C 4}^{\prime}$ & 1,34 & 39,01 & 0,99 & 40,00 \\
\hline & 1,52 & I & 1,55 & I \\
\hline Cs' & 1,61 & 24,81 & 1,72 & 25,12 \\
\hline C6' & 0,94 & 21,87 & 0,96 & 21,69 \\
\hline $\mathrm{C}^{\prime}$ & 0,95 & 24,45 & 0,91 & 24,20 \\
\hline Boc-CO & I & 155,60 & I & 156,35 \\
\hline Boc-C(iv) & I & 79,60 & I & 79,60 \\
\hline Boc-CH3 & 1,19 & 28,37 & 1,43 & 28,64 \\
\hline CH2 (2'-ethyl) & 1,84 & 28,53 & 1,83 & 29,56 \\
\hline & 1,85 & I & 1,83 & I \\
\hline CH3 (2'-ethyl) & 0,95 & 8,43 & 0,84 & 7,94 \\
\hline
\end{tabular}

Table S2: $:{ }^{1} \mathrm{H}$ and ${ }^{13} \mathrm{C}$ c.s. values of the epimeric pair $\mathbf{4 a , b}$

\begin{tabular}{|c|c|c|c|c|}
\hline & \multicolumn{2}{|c|}{ Minor (4a) } & \multicolumn{2}{|c|}{ Major (4b) } \\
\hline & ${ }^{1} \mathrm{H}$ NMR & ${ }^{13} \mathrm{C}$ NMR & ${ }^{1} \mathrm{H}$ NMR & ${ }^{13} \mathrm{C}$ NMR \\
\hline C1 & I & 79,76 & I & 79,59 \\
\hline $\mathrm{C}_{2}$ & 5,68 & 75,28 & 5,65 & 75,24 \\
\hline $\mathrm{C2}(\mathrm{CO})$ & I & 167,43 & 1 & 167,38 \\
\hline C2 (C(iv)) & I & 129,47 & I & 129,45 \\
\hline C2 (C-orto) & 8,05 & 130,33 & 8,08 & 130,48 \\
\hline C2 (C-meta) & 7,43 & 128,88 & 7,45 & 128,88 \\
\hline C2 (C-para) & 7,58 & 134,01 & 7,57 & 133,93 \\
\hline $\mathrm{C3}$ & 3,82 & 46,97 & 3,80 & 46,90 \\
\hline $\mathrm{C} 4$ & I & 81,20 & I & 81,27 \\
\hline C4 (Acetyl-CO) & I & 171,01 & / & 171,16 \\
\hline $\mathrm{C4}$ (Acetyl-CH3) & 2,43 & 22,81 & 2,50 & 22,81 \\
\hline C5 & 4,90 & 84,65 & 4,90 & 84,63 \\
\hline C6 & 1,87 & 37,45 & 1,86 & 37,48 \\
\hline & 2,50 & I & 2,49 & I \\
\hline C7 & 4,46 & 72,50 & 4,42 & 72,53 \\
\hline $\mathrm{C} 7$ (CH2-TES) & 0,56 & 5,54 & 0,55 & 5,52 \\
\hline C7 (CH3-TES) & 0,90 & 7,03 & 0,89 & 7,02 \\
\hline C8 & I & 58,64 & I & 58,69 \\
\hline C9 & I & 202,05 & I & 202,04 \\
\hline C10 & 6,44 & 75,15 & 6,42 & 75,13 \\
\hline C10 (Acetyl-CO) & I & 169,57 & 1 & 169,53 \\
\hline C10 (Acetyl-CH3) & 2,16 & 21,18 & 2,16 & 21,15 \\
\hline C11 & I & 133,50 & 1 & 133,47 \\
\hline $\mathrm{C} 12$ & I & 140,62 & I & 140,43 \\
\hline $\mathrm{Cl3}$ & 6,19 & 72,07 & 6,42 & 71,82 \\
\hline $\mathrm{C} 14$ & 2,21 & 36,07 & 2,02 & 36,04 \\
\hline & 2,23 & I & 2,26 & I \\
\hline $\mathrm{C15}$ & 1 & 43,69 & 1 & 43,62 \\
\hline C16 & 1,22 & 22,00 & 1,21 & 22,04 \\
\hline C17 & 1,24 & 26,95 & 1,23 & 27,00 \\
\hline C18 & 2,07 & 15,14 & 2,03 & 15,35 \\
\hline C19 & 1,67 & 10,43 & 1,67 & 10,40 \\
\hline C20 & 4,13 & 76,86 & 4,14 & 77,00 \\
\hline & 4,29 & I & 4,28 & I \\
\hline $\mathrm{Cl}^{\prime}$ & I & 174,07 & I & 175,35 \\
\hline $\mathrm{C}^{\prime}$ & 1 & 84,65 & 1 & 84,07 \\
\hline $\mathrm{C}^{\prime}$ & 4,09 & 53,78 & 4,07 & 52,66 \\
\hline C4 & 1,08 & 40,95 & 1,32 & 39,16 \\
\hline & 1,59 & I & 1,44 & I \\
\hline C5' $^{\prime}$ & 1,60 & 25,09 & 1,60 & 24,78 \\
\hline C6' & 0,94 & 21,70 & 0,96 & 21,78 \\
\hline C7' & 0,92 & 24,26 & 0,93 & 24,46 \\
\hline Boc-CO & I & 156,70 & I & 155,79 \\
\hline Boc-C(iv) & l & 80,05 & / & 79,66 \\
\hline Boc-CH3 & 1,39 & 28,61 & 1,25 & 28,42 \\
\hline CH (2'-isopropyl) & 2,11 & 35,06 & 2,08 & 34,25 \\
\hline CH3 (2'-isopropyl) & 0,96 & 17,20 & 0,96 & 17,19 \\
\hline CH3 (2'-isopropyl) & 1,04 & 18,01 & 1,07 & 17,47 \\
\hline
\end{tabular}


Table S3: ${ }^{1} \mathrm{H}$ and ${ }^{13} \mathrm{C}$ c.s. values of the epimeric pair 5a,b

\begin{tabular}{|c|c|c|c|c|}
\hline & \multicolumn{2}{|c|}{$5 a$} & \multicolumn{2}{|c|}{$5 b$} \\
\hline & ${ }^{1} \mathrm{H}$ NMR & ${ }^{13} \mathrm{C}$ NMR & ${ }^{1} \mathrm{H}$ NMR & ${ }^{13} \mathrm{C}$ NMR \\
\hline $\mathrm{Cl}$ & I & 79,68 & l & 79,93 \\
\hline $\mathrm{C}_{2}$ & 5,66 & 75,19 & 5,68 & 75,25 \\
\hline $\mathrm{C2}(\mathrm{CO})$ & I & 167,41 & I & 167,32 \\
\hline C2 (C(iv)) & I & 129,45 & I & 129,52 \\
\hline C2 (C-orto) & 8,10 & 130,55 & 8,03 & 130,24 \\
\hline C2 (C-meta) & 7,45 & 128,90 & 7,41 & 128,89 \\
\hline C2 (C-para) & 7,58 & 133,95 & 7,57 & 134,01 \\
\hline C3 & 3,83 & 46,93 & 3,83 & 47,02 \\
\hline $\mathrm{C} 4$ & I & 81,39 & I & 81,35 \\
\hline C4 (Acetyl-CO) & l & 171,16 & I & 170,95 \\
\hline $\mathrm{C} 4$ (Acetyl-CH3) & 2,49 & 22,81 & 2,42 & 22,98 \\
\hline C5 & 4,90 & 84,66 & 4,88 & 84,68 \\
\hline Co & 1,88 & 37,56 & 1,88 & 37,42 \\
\hline & 2,51 & I & 2,49 & I \\
\hline C7 & 4,44 & 72,53 & 4,47 & 72,50 \\
\hline $\mathrm{C} 7$ ( $\mathrm{CH} 2-\mathrm{TES})$ & 0,56 & 5,52 & 0,56 & 5,55 \\
\hline $\mathrm{C} 7$ (CH3-TES) & 0,90 & 7,05 & 0,91 & 7,04 \\
\hline $\mathrm{C} 8$ & I & 58,73 & I & 58,62 \\
\hline C9 & I & 202,09 & l & 201,91 \\
\hline $\mathrm{Cl0}$ & 6,44 & 75,22 & 6,44 & 75,09 \\
\hline C10 (Acetyl-Co) & I & 169,54 & I & 169,61 \\
\hline $\mathrm{C} 10$ (Acetyl-CH3) & 2,16 & 21,17 & 2,16 & 21,18 \\
\hline C11 & I & 133,35 & I & 133,54 \\
\hline C12 & I & 140,74 & I & 140,35 \\
\hline C13 & 6,44 & 71,16 & 6,17 & 72,11 \\
\hline C14 & 2,04 & 36,03 & 2,18 & 36,13 \\
\hline & 2,26 & I & 2,23 & I \\
\hline C15 & 1 & 43,70 & I & 43,65 \\
\hline $\mathrm{Cl6}$ & 1,22 & 21,95 & 1,23 & 22,07 \\
\hline C17 & 1,24 & 26,93 & 1,24 & 26,92 \\
\hline $\mathrm{C} 18$ & 2,05 & 15,27 & 2,10 & 15,16 \\
\hline C19 & 1,68 & 10,42 & 1,69 & 10,48 \\
\hline C20 & 4,15 & 77,00 & 4,15 & 76,84 \\
\hline & 4,29 & I & 4,26 & I \\
\hline C1' & 1 & 174,76 & 1 & 174,01 \\
\hline C2' & I & 85,52 & I & 85,40 \\
\hline C3' & 4,14 & 53,91 & 4,27 & 52,28 \\
\hline C4 & 1,08 & 40,70 & 0,93 & 42,77 \\
\hline & 1,58 & I & 1,57 & I \\
\hline C5 & 1,56 & 25,04 & 1,65 & 24,89 \\
\hline C6 & 0,97 & 21,86 & 0,92 & 21,75 \\
\hline C7! & 0,94 & 24,61 & 0,90 & 24,43 \\
\hline Boc-CO & I & 155,86 & I & 155,45 \\
\hline Boc-C(iv) & I & 79,66 & I & 79,77 \\
\hline Boc-CH3 & 1,30 & 28,47 & 1,43 & 28,74 \\
\hline $\mathbf{C}\left(2^{\prime}\right.$-terzbutyl) & I & 38,15 & 1 & 39,26 \\
\hline $\mathrm{CH} 3\left(2^{\prime}\right.$-terzbutyl) & 1,09 & 27,05 & 1,07 & 26,96 \\
\hline
\end{tabular}

a) only major conformers of both 2'-epimers are reported. See table S22 for c.s. values of both minor conformers.
Table S4: $:{ }^{1} \mathrm{H}$ and ${ }^{13} \mathrm{C}$ c.s. values of the epimeric pair $\mathbf{6 a , b}$

\begin{tabular}{|c|c|c|c|c|}
\hline & \multicolumn{2}{|c|}{$6 \mathbf{a}$} & \multicolumn{2}{|c|}{$6 b$} \\
\hline & ${ }^{1} \mathrm{H}$ NMR & ${ }^{13} \mathrm{C}$ NMR & ${ }^{1} \mathrm{H}$ NMR & ${ }^{13} \mathrm{C}$ NMR \\
\hline $\mathrm{Cl}$ & l & 79,25 & l & 79,20 \\
\hline $\mathrm{C} 2$ & 5,63 & 75,03 & 5,63 & 74,97 \\
\hline $\mathrm{C2}(\mathrm{CO})$ & I & 167,34 & I & 167,29 \\
\hline C2 (C(iv)) & I & 129,44 & I & 129,46 \\
\hline C2 (C-orto) & 8,10 & 130,55 & 8,08 & 130,39 \\
\hline C2 (C-meta) & 7,44 & 128,85 & 7,47 & 128,92 \\
\hline C2 (C-para) & 7,56 & 133,79 & 7,60 & 134,07 \\
\hline $\begin{array}{r}\mathrm{C} 3 \\
\end{array}$ & 3,75 & 46,98 & 3,72 & 47,02 \\
\hline $\mathrm{C} 4$ & I & 81,25 & I & 81,49 \\
\hline C4 (Acetyl-CO) & I & 171,23 & I & 171,36 \\
\hline C4 (Acetyl-CH3) & 2,48 & 22,94 & 2,41 & 22,76 \\
\hline C5 & 4,93 & 84,62 & 4,92 & 84,44 \\
\hline C6 & 1,88 & 37,55 & 1,87 & 37,64 \\
\hline & 2,52 & I & 2,51 & I \\
\hline C7 & 4,41 & 72,69 & 4,35 & 72,67 \\
\hline C7 (CH2-TES) & 0,54 & 5,54 & 0,53 & 5,54 \\
\hline C7 (CH3-TES) & 0,90 & 7,01 & 0,88 & 7,03 \\
\hline C8 & I & 58,76 & I & 58,95 \\
\hline C9 & 1 & 202,13 & I & 202,14 \\
\hline C10 & 6,34 & 75,22 & 6,32 & 75,31 \\
\hline C10 (Acetyl-CO) & I & 169,43 & I & 169,51 \\
\hline $\mathrm{C10}$ (Acetyl-CH3) & 2,12 & 21,11 & 2,11 & 21,12 \\
\hline C11 & I & 133,44 & I & 133,55 \\
\hline $\mathrm{C} 12$ & I & 140,86 & I & 140,80 \\
\hline C13 & 6,34 & 72,20 & 6,08 & 71,68 \\
\hline C14 & 2,06 & 35,75 & 2,15 & 35,96 \\
\hline & 2,27 & I & 2,28 & / \\
\hline $\mathrm{C} 15$ & 1 & 43,47 & I & 43,48 \\
\hline C16 & 1,18 & 27,03 & 1,14 & 26,97 \\
\hline $\mathrm{C} 17$ & 1,18 & 21,71 & 1,18 & 21,19 \\
\hline $\mathrm{C} 18$ & 1,67 & 14,57 & 1,58 & 14,56 \\
\hline C19 & 1,68 & 10,39 & 1,67 & 10,33 \\
\hline C20 & 4,15 & 77,02 & 4,16 & 77,00 \\
\hline & 4,26 & I & 4,29 & I \\
\hline C1' & 1 & 174,50 & 1 & 172,50 \\
\hline C2 & / & 82,43 & I & 83,15 \\
\hline $\mathbf{C}^{\prime}$ & 4,47 & 55,59 & 4,47 & 56,24 \\
\hline C4 & 0,96 & 38,33 & 1,17 & 40,13 \\
\hline & 1,45 & / & 1,75 & l \\
\hline C5! & 1,55 & 24,86 & $?$ & 25,39 \\
\hline C6 & 0,80 & 21,71 & 0,98 & 21,72 \\
\hline C7 & 0,82 & 24,13 & 0,94 & 24,17 \\
\hline Boc-CO & I & 155,66 & I & 156,37 \\
\hline Boc-C(iv) & I & 79,68 & I & 79,75 \\
\hline Boc-CH3 & 1,23 & 28,39 & 1,19 & 28,36 \\
\hline C2' (C(iv)-aryl) & I & 138,64 & I & 139,99 \\
\hline C2' (C(orto)-aryl) & 7,69 & 126,31 & 7,63 & 125,86 \\
\hline C2' (C(meta)-aryl) & 7,41 & 128,70 & 7,33 & 128,51 \\
\hline C2' (C(para)-aryl) & 7,33 & 128,57 & 7,27 & 128,48 \\
\hline
\end{tabular}


Table S5: ${ }^{1} \mathrm{H}$ and ${ }^{13} \mathrm{C}$ c.s. values of the epimeric pair 7a,b

\begin{tabular}{|c|c|c|c|c|}
\hline & \multicolumn{2}{|c|}{$7 \mathbf{a}$} & \multicolumn{2}{|c|}{$7 \mathbf{b}$} \\
\hline & IH NMR & ${ }^{13} \mathrm{C}$ NMR & ${ }^{1} \mathrm{H}$ NMR & ${ }^{13} \mathrm{C}$ NMR \\
\hline $\mathrm{Cl}$ & I & 79,36 & I & 79,32 \\
\hline $\mathrm{C}_{2}$ & 5,63 & 75,31 & 5,67 & 75,12 \\
\hline $\mathrm{C2}(\mathrm{CO})$ & I & 167,38 & I & 167,30 \\
\hline C2 (C(iv)) & I & 129,60 & I & 129,49 \\
\hline C2 (C-orto) & 8,12 & 130,61 & 8,06 & 130,37 \\
\hline C2 (C-meta) & 7,44 & 128,89 & 7,45 & 128,90 \\
\hline C2 (C-para) & 7,55 & 133,71 & 7,58 & 134,00 \\
\hline C3 & 3,78 & 46,84 & 3,79 & 46,95 \\
\hline C4 & I & 81,22 & 1 & 81,74 \\
\hline C4 (Acetyl-Co) & I & 170,89 & I & 170,96 \\
\hline $\mathrm{C} 4$ (Acetyl-CH3) & 2,54 & 23,27 & 2,44 & 22,92 \\
\hline C5 & 4,91 & 84,56 & 4,91 & 84,56 \\
\hline C6 & 1,87 & 37,56 & 1,89 & 37,57 \\
\hline & 2,49 & I & 2,50 & I \\
\hline c7 & 4,42 & 72,52 & 4,43 & 72,67 \\
\hline C7 (CH2-TES) & 0,54 & 5,55 & 0,55 & 5,58 \\
\hline C7 (CH3-TES) & 0,89 & 7,02 & 0,89 & 7,02 \\
\hline & I & 58,69 & I & 58,86 \\
\hline & I & 202,03 & I & 202,05 \\
\hline C10 & 6,39 & 75,17 & 6,42 & 75,27 \\
\hline C10 (Acetyl-CO) & I & 169,43 & I & 169,53 \\
\hline C10 (Acetyl-CH3) & 2,13 & 21,13 & 2,15 & 21,13 \\
\hline $\mathrm{C} 11$ & 1 & 133,71 & I & 133,76 \\
\hline C12 & I & 140,79 & I & 140,43 \\
\hline C13 & 6,30 & 72,50 & 6,21 & 72,45 \\
\hline C14 & 2,11 & 35,72 & 2,19 & 35,82 \\
\hline & 2,35 & I & 2,20 & I \\
\hline C15 & I & 43,58 & 1 & 43,64 \\
\hline C16 & 1,18 & 26,97 & 1,19 & 27,00 \\
\hline C17 & 1,18 & 21,87 & 1,21 & 21,59 \\
\hline C18 & 1,90 & 14,45 & 2,02 & 15,14 \\
\hline C19 & 1,67 & 10,42 & 1,68 & 10,36 \\
\hline C20 & 4,17 & 76,92 & 4,15 & 77,00 \\
\hline & 4,25 & / & 4,28 & I \\
\hline cil & 1 & 174,72 & l & 173,48 \\
\hline $\mathrm{C}^{\prime}$ & I & 81,54 & 1 & 81,34 \\
\hline $\mathrm{C}^{\prime}$ & 4,16 & 53,72 & 4,15 & 54,43 \\
\hline C4: & 1,26 & 38,09 & 1,03 & 39,50 \\
\hline & 1,41 & I & 1,54 & I \\
\hline C5' & 1,57 & 24,81 & 1,68 & 25,26 \\
\hline C6 & 0,88 & 21,89 & 0,93 & 21,73 \\
\hline C7 & 0,90 & 24,22 & 0,90 & 24,06 \\
\hline Boc-CO & I & 155,54 & I & 156,37 \\
\hline Boc-C(iv) & I & 79,60 & I & 79,60 \\
\hline Boc-CH3 & 1,21 & 28,39 & 1,38 & 28,59 \\
\hline CH-vinyl & 6,05 & 135,58 & 6,07 & 137,41 \\
\hline CH2-vinyl & 5,39 & 118,26 & 5,28 & 117,12 \\
\hline & 5,62 & 1 & 5,54 & I \\
\hline
\end{tabular}


Table S6: Full NMR data for the library of 2 -substituted taxanes, including measured ${ }^{2,3} \mathrm{~J}_{\mathrm{HC}}$, ROESY, TAD and RI values. The intensity of dipolar effects (ROESY) is expressed in terms of three categories: $s=$ strong, $m=$ medium, $w=$ weak.

\begin{tabular}{|c|c|c|c|c|c|c|c|c|c|}
\hline \multirow[b]{2}{*}{$\mathrm{R}$} & \multicolumn{2}{|c|}{ Conformers } & \multicolumn{3}{|c|}{ Coupling constants $(\mathrm{Hz})$} & \multirow{2}{*}{$\begin{array}{c}\text { Spatial relationship } \\
\text { ROESY }\end{array}$} & \multirow[b]{2}{*}{$\begin{array}{c}\text { Database } \\
\text { element }\end{array}$} & \multirow[b]{2}{*}{ TAD } & \multirow[b]{2}{*}{$\begin{array}{c}\text { RI } \\
(\% 00)\end{array}$} \\
\hline & c2'-C3'syn & i-Bu $\overbrace{\substack{\mathrm{NHBOc} \\
\mathrm{C} 2^{\prime}-\mathrm{C} 3^{\prime} \text { anti }}}^{\mathrm{COOR}}$ & ${ }^{3} J_{\mathrm{H}^{3}} \cdot \mathrm{C}_{1}$ & ${ }^{3} J_{H_{3}} \cdot-C_{R}$ & ${ }^{2} J_{\mathrm{H}^{3}}, \mathrm{C}_{2}$ & & & & \\
\hline \multirow{4}{*}{ مs } & Minor & \multirow{2}{*}{ I } & \multirow{2}{*}{$<1,4$} & \multirow{2}{*}{$<1,4$} & \multirow{2}{*}{$<1,4$} & \multirow{2}{*}{$\begin{array}{c}\mathrm{H}_{3}{ }^{\prime}-\mathrm{CH}_{3}(\mathrm{Et}) s ; \mathrm{CH}_{2}(\mathrm{Et})-\mathrm{C}_{4}{ }^{\prime} \mathrm{H} s ; \mathrm{CH}_{2}(\mathrm{Et})- \\
\mathrm{C}_{7}{ }^{\prime} \mathrm{H} s ; \mathrm{H}_{3}{ }^{\prime}-\mathrm{CH}_{2}(\mathrm{Et}) m ; \mathrm{OH}-\mathrm{C}_{7}{ }^{\prime} \mathrm{H} w\end{array}$} & syn & 1,20 & \multirow{2}{*}{386} \\
\hline & (3a) & & & & & & anti & 5,83 & \\
\hline & \multirow{2}{*}{ I } & \multirow{2}{*}{$\begin{array}{c}\text { Major } \\
(\mathbf{3 b})\end{array}$} & \multirow{2}{*}{$<1,0$} & \multirow{2}{*}{$<1,0$} & \multirow{2}{*}{$<1,0$} & \multirow{2}{*}{$\begin{array}{c}\mathrm{H}_{3}{ }^{\prime}-\mathrm{CH}_{2}(\mathrm{Et}) s ; \mathrm{NH}-\mathrm{CH}_{2}(\mathrm{Et}) s ; \mathrm{CH}_{2}(\mathrm{Et})- \\
\mathrm{CH}_{3}(\mathrm{Boc}) w\end{array}$} & syn & 5,83 & \multirow{2}{*}{386} \\
\hline & & & & & & & anti & 1,20 & \\
\hline & \multirow{2}{*}{ I } & \multirow{2}{*}{$\begin{array}{l}\text { Minor } \\
(\mathbf{4 a})\end{array}$} & \multirow{2}{*}{2,4} & \multirow{2}{*}{$<1,2$} & \multirow{2}{*}{$<1,2$} & \multirow{2}{*}{$\begin{array}{c}\mathrm{H}_{3}{ }^{\prime}-\mathrm{CH}(\mathrm{i}-\mathrm{Pr}) s ; \\
\mathrm{CH} \text { (i-Pr) } m ; \mathrm{NH}-\mathrm{CH} w ;\end{array}$} & syn & 7,24 & \multirow{2}{*}{783} \\
\hline & & & & & & & anti & 0,82 & \\
\hline & \multirow{2}{*}{$\begin{array}{c}\text { Major } \\
(\mathbf{4 b})\end{array}$} & \multirow{2}{*}{ l } & \multirow{2}{*}{$<0,5$} & \multirow{2}{*}{$<0,5$} & \multirow{2}{*}{$<0,5$} & $\mathrm{CH}(\mathrm{i}-\mathrm{Pr})-\mathrm{CH}_{2}(\mathrm{i}-\mathrm{Bu}) s ; \mathrm{CH}(\mathrm{i}-\mathrm{Pr})-\mathrm{CH}_{3}(\mathrm{i}-$ & syn & 0,82 & 783 \\
\hline & & & & & & Bu) $s ; \mathrm{H}_{3}{ }^{\prime}-\mathrm{CH}(\mathrm{i}-\mathrm{Pr}) m ; \mathrm{H}_{3}{ }^{\prime}-\mathrm{CH}_{3}(\mathrm{i}-\mathrm{Pr}) w$; & anti & 7,24 & 183 \\
\hline & $5 \mathrm{a}^{\mathrm{a}}$ & 1 & $1 ?$ & $1 ?$ & 110 & $\mathrm{CH}_{3}(\mathrm{t}-\mathrm{Bu})-\mathrm{H}_{3}{ }^{\prime} s ; \mathrm{CH}_{3}(\mathrm{t}-\mathrm{Bu})-\mathrm{CH}_{2}(\mathrm{i}-\mathrm{Bu}) s ;$ & syn & 2,88 & 05 \\
\hline & Ja & 1 & 1,2 & 1,2 & 1,2 & $\mathrm{H}(\mathrm{C} 13)-\mathrm{CH}_{3}(\mathrm{Boc}) m ; \mathrm{H}(\mathrm{C} 13)-\mathrm{H}_{3}{ }^{\prime} w$ & anti & 5,58 & 93 \\
\hline & 1 & $5 \mathbf{b}$ & $<07$ & $<07$ & $<07$ & $\mathrm{H}_{3}{ }^{\prime}-\mathrm{CH}_{3}(\mathrm{t}-\mathrm{Bu}) s ; \mathrm{NH}-\mathrm{CH}_{3}(\mathrm{t}-\mathrm{Bu}) m$ & syn & 5,58 & 05 \\
\hline & & & 0,1 & 0,1 & 0,1 & $\mathrm{H}(\mathrm{C} 13)-\mathrm{H}_{3}, w ; \mathrm{H}(\mathrm{C} 13)-\mathrm{CH}_{2}(\mathrm{i}-\mathrm{Bu}) w$ & anti & 2,88 & 93 \\
\hline & 6 & 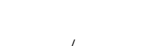 & -60 & 600 & 500 & $\mathrm{H}_{\text {orto }}-\mathrm{H}_{3}, s ; \mathrm{H}_{\text {orto }}-$ i-butile $m ;$ & syn & 1,49 & 200 \\
\hline & $\mathbf{6 a}$ & 1 & $<0.9$ & $<0.9$ & $<0.9$ & $\mathrm{H}_{\text {orto }}-\mathrm{NH} w$ & anti & 7,29 & 389 \\
\hline & 1 & $6 \mathbf{b}$ & $<1.0$ & $<10$ & $<10$ & $\mathrm{H}_{\text {orto }}-\mathrm{H}_{3}, s ; \mathrm{H}_{\text {orto }}-\mathrm{NH} m ; \mathrm{H}_{\text {orto }}-\mathrm{CH}_{3}(\mathrm{Boc})$ & syn & 7,29 & 389 \\
\hline & & & & & & $m$ & anti & 1,49 & \\
\hline & $7 a$ & 1 & $<10$ & $<10$ & $<10$ & $\mathrm{CH}$ (vinyl)-i-Bu $m$; & syn & 1,24 & 448 \\
\hline & & & 1,0 & 1,0 & 1,0 & $\mathrm{OH}$ vs $\mathrm{NH} w$ & anti & 6,80 & 440 \\
\hline & & & & & & $\mathrm{CH}\left(\right.$ vinyl) $-\mathrm{H}_{3}, s$; & syn & 6,80 & \\
\hline & 1 & 10 & $<, 0$ & 1,0 & 1,0 & $\mathrm{CH}$ (vinyl)- $\mathrm{NH} m$ & anti & 1,24 & 448 \\
\hline
\end{tabular}

a) Spectra acquired in DMSO-d6 at $50^{\circ} \mathrm{C}$ 


\section{2'-Ethyltaxanes:}

Original and modified UDB analysis when epimeric 2'-methyltaxanes are set as database elements

Table S7: Side chain ${ }^{13} \mathrm{C}$ NMR c.s. values of the epimeric 2'-methyl taxanes and the compounds under investigation

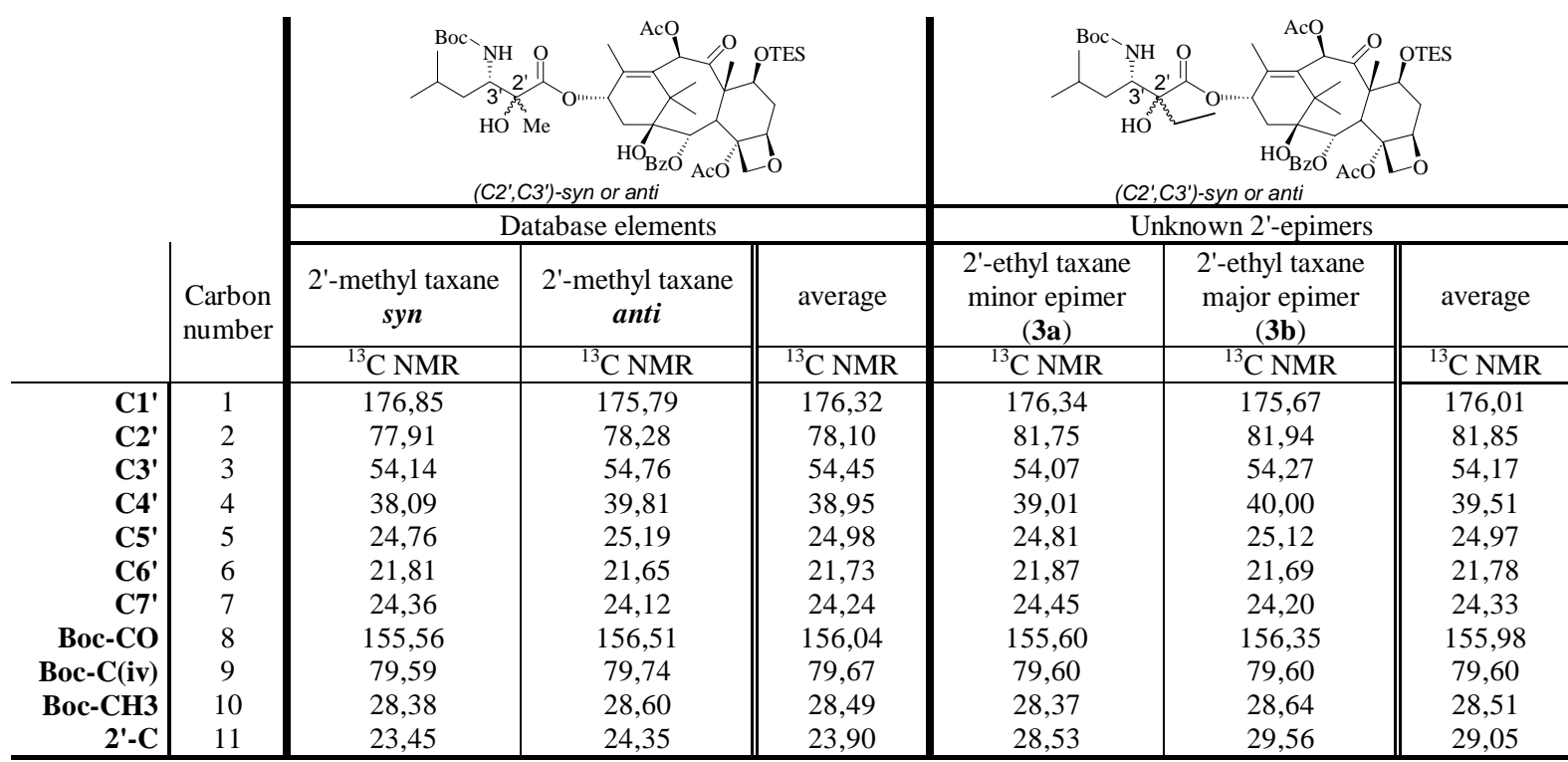

\section{Original UBD analysis}

Table S8: Original UDB analysis performed on title compound

\begin{tabular}{|c|c|c|c|c|c|c|c|c|c|c|}
\hline \multirow{4}{*}{$\begin{array}{l}\text { Carbon } \\
\text { number }\end{array}$} & \multicolumn{2}{|c|}{ Calculated c.s. ${ }^{a}$} & \multirow[b]{3}{*}{$\begin{array}{l}\text { Correcting } \\
\text { factor }^{\mathrm{b}}\end{array}$} & \multicolumn{2}{|c|}{ Adjusted c.s. ${ }^{\mathrm{c}}$} & \multicolumn{5}{|c|}{ Chemical shift difference } \\
\hline & \multirow[b]{2}{*}{$\begin{array}{l}\text { 2'methyl } \\
\text { taxane }\end{array}$} & \multirow[b]{2}{*}{$\begin{array}{l}\text { 2'ethyl } \\
\text { taxane }\end{array}$} & & \multirow{2}{*}{$\begin{array}{c}2^{\prime} \text {-ethyl } \\
\text { taxane } \\
\text { minor } \\
\text { epimer } \\
(\mathbf{3 a}) \\
\end{array}$} & \multirow{2}{*}{$\begin{array}{c}\text { '-ethyl } \\
\text { taxane } \\
\text { major } \\
\text { epimer } \\
(\mathbf{3 b}) \\
\end{array}$} & \multicolumn{5}{|c|}{ (2'-methyl taxane) - (adjusted 2'-ethyl taxane) } \\
\hline & & & & & & syn-3a & anti-3a & & $s y n-3 b$ & $a n t i-3 b$ \\
\hline & $\begin{array}{c}{ }^{13} \mathrm{C} \\
\mathrm{NMR}\end{array}$ & $\begin{array}{c}{ }^{13} \mathrm{C} \\
\text { NMR }\end{array}$ & ${ }^{13} \mathrm{C}$ NMR & $\begin{array}{c}{ }^{13} \mathrm{C} \\
\text { NMR }\end{array}$ & $\begin{array}{c}{ }^{13} \mathrm{C} \\
\mathrm{NMR}\end{array}$ & ${ }^{13} \mathrm{C}$ NMR & ${ }^{13} \mathrm{C}$ NMR & & ${ }^{13} \mathrm{C}$ NMR & ${ }^{13} \mathrm{C} \mathrm{NMR}$ \\
\hline 1 & 174,50 & 176,00 & $-1,50$ & 174,84 & 174,17 & 2,01 & 0,95 & & 2,68 & 1,62 \\
\hline 2 & 87,90 & 90,80 & $-2,90$ & 78,85 & 79,04 & $-0,94$ & $-0,57$ & & $-1,13$ & $-0,76$ \\
\hline 3 & 55,30 & 52,80 & 2,50 & 56,57 & 56,77 & $-2,43$ & $-1,81$ & & $-2,63$ & $-2,01$ \\
\hline 4 & 36,10 & 36,40 & $-0,30$ & 38,71 & 39,70 & $-0,62$ & 1,10 & & $-1,61$ & 0,11 \\
\hline 5 & 23,70 & 23,70 & 0,00 & 24,81 & 25,12 & $-0,05$ & 0,38 & & $-0,36$ & 0,07 \\
\hline 6 & 22,30 & 22,30 & 0,00 & 21,87 & 21,69 & $-0,06$ & $-0,22$ & & 0,12 & $-0,04$ \\
\hline 7 & 22,30 & 22,30 & 0,00 & 24,45 & 24,20 & $-0,09$ & $-0,33$ & & 0,16 & $-0,08$ \\
\hline 8 & 157,20 & 157,20 & 0,00 & 155,60 & 156,35 & $-0,04$ & 0,91 & & $-0,79$ & 0,16 \\
\hline 9 & 70,60 & 70,60 & 0,00 & 79,60 & 79,60 & $-0,01$ & 0,14 & & $-0,01$ & 0,14 \\
\hline 10 & 28,70 & 28,70 & 0,00 & 28,37 & 28,64 & 0,01 & 0,23 & & $-0,26$ & $-0,04$ \\
\hline 11 & 17,90 & 24,40 & $-6,50$ & 22,03 & 23,06 & 1,42 & 2,32 & & 0,39 & 1,29 \\
\hline & & & & & & 7,68 & 8,96 & $\mathbf{T A D}^{\mathrm{d}}$ & 10,14 & 6,32 \\
\hline
\end{tabular}

a) CS ChemNMR Pro version 1.0, Renate Buergin Schaller, Development Centre, Bergstr. 114, Zurich, Switzerland, installed in CS ChemDraw Pro version 7.0, was used.

b) Correcting factor $=($ database element c.s. calculated value $)-($ unknown 2 '-epimer c.s. calculated value $)$

c) adjusted c.s. value $=($ unknown 2 -epimer c.s. measured value $)+($ correcting factor $)$

d) $\mathrm{TAD}=$ Total Absolute Deviation: sum of absolute values of all c.s. differences $=\Sigma_{\mathrm{i}}\left|\Delta \delta_{\mathrm{i}}\right|$ 


\section{Modified UDB approach}

Table S9: $\quad$ Modified UDB analysis performed on title compound.

\begin{tabular}{|c|c|c|c|c|c|c|c|c|}
\hline & \multirow{3}{*}{$\begin{array}{l}\text { Correcting } \\
\text { factor }^{\mathrm{a}}\end{array}$} & \multicolumn{2}{|c|}{ Adjusted c.s. values ${ }^{b}$} & \multirow{2}{*}{\multicolumn{5}{|c|}{$\frac{\text { Chemical shift difference } \Delta \delta_{\mathrm{i}}}{\text { (2'-ethyl taxane) }-\left(2^{\prime} \text {-methyl taxane }\right)}$}} \\
\hline & & \multirow{2}{*}{$\begin{array}{l}\text { 2'-ethyl } \\
\text { taxane } \\
\text { minor } \\
\text { epimer } \\
\text { (3a) }\end{array}$} & \multirow{2}{*}{$\begin{array}{l}\text { ''-ethyl } \\
\text { taxane } \\
\text { major } \\
\text { epimer } \\
(\mathbf{3 b}) \\
\end{array}$} & & & & & \\
\hline & & & & 3a-syn & 3a-anti & & $3 b-s y n$ & $3 b-a n t i$ \\
\hline $\begin{array}{l}\text { Carbon } \\
\text { number }\end{array}$ & ${ }^{13} \mathrm{C}$ NMR & ${ }^{13} \mathrm{C}$ NMR & ${ }^{13} \mathrm{C}$ NMR & ${ }^{13} \mathrm{C}$ NMR & ${ }^{13} \mathrm{C}$ NMR & & ${ }^{13} \mathrm{C}$ NMR & ${ }^{13} \mathrm{C}$ NMR \\
\hline 1 & $-0,31$ & 176,66 & 175,99 & $-0,19$ & 0,87 & & $-0,87$ & 0,19 \\
\hline 2 & 3,75 & 78,00 & 78,19 & 0,09 & $-0,28$ & & 0,28 & $-0,09$ \\
\hline 3 & $-0,28$ & 54,35 & 54,55 & 0,21 & $-0,41$ & & 0,41 & $-0,21$ \\
\hline 4 & 0,55 & 38,46 & 39,45 & 0,37 & $-1,36$ & & 1,36 & $-0,36$ \\
\hline 5 & $-0,01$ & 24,82 & 25,13 & 0,06 & $-0,37$ & & 0,37 & $-0,06$ \\
\hline 6 & 0,05 & 21,82 & 21,64 & 0,01 & 0,17 & & $-0,17$ & $-0,01$ \\
\hline 7 & 0,08 & 24,37 & 24,12 & 0,01 & 0,25 & & $-0,24$ & 0,00 \\
\hline 8 & $-0,06$ & 155,66 & 156,41 & 0,10 & $-0,85$ & & 0,85 & $-0,10$ \\
\hline 9 & $-0,06$ & 79,67 & 79,67 & 0,07 & $-0,08$ & & 0,07 & $-0,08$ \\
\hline 10 & 0,02 & 28,36 & 28,63 & $-0,02$ & $-0,25$ & & 0,25 & 0,02 \\
\hline 11 & 5,15 & 23,39 & 24,42 & $-0,07$ & $-0,97$ & & 0,96 & 0,06 \\
\hline & & & & 1,20 & 5,83 & TAD $^{\mathrm{c}}$ & 5,83 & 1,20 \\
\hline
\end{tabular}

a) correcting factor: $\mathrm{CF}_{\mathrm{i}}=\mathrm{m}_{\mathrm{i}}^{\text {epimers }}-\mathrm{m}_{\mathrm{i}}{ }^{\mathrm{DB}}$, where $\mathrm{m}_{\mathrm{i}}$ 's are mean chemical shift values

b) adjusted chemical shift values, obtained $c . s_{\cdot \mathrm{i}}=\mathrm{c} . \mathrm{s}_{{ }_{\mathrm{i}}}-\mathrm{CF}_{\mathrm{i}}$

c) $\mathrm{TAD}=$ Total Absolute Deviation: sum of absolute values of all c.s. differences $=\Sigma_{\mathrm{i}}\left|\Delta \delta_{\mathrm{i}}\right|$

Minor epimer (3a): $\quad$ Syn

Major epimer (3b): Anti 
Modified UDB analysis when epimeric methyl 2-methylisoserinate are set as database elements

Table S10: Side chain ${ }^{13} \mathrm{C}$ NMR c.s. values of epimeric 2'-methyl isoserinate and compound in study

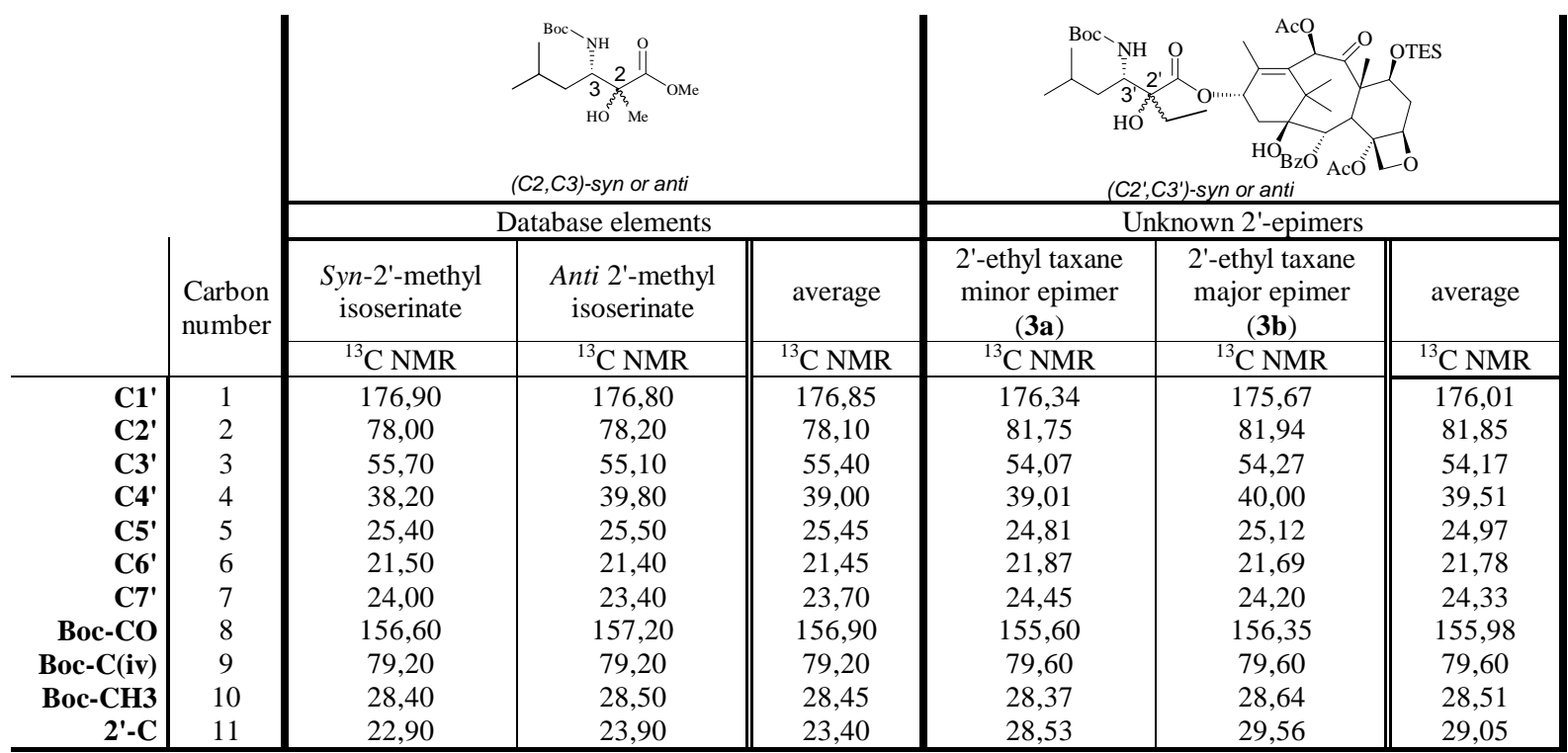

\section{Modified UDB approach}

Table S11: Modified UDB analysis performed on title compound.

\begin{tabular}{|c|c|c|c|c|c|c|c|c|}
\hline & \multirow{3}{*}{$\begin{array}{l}\text { Correcting } \\
\text { factor }^{\mathrm{a}}\end{array}$} & \multicolumn{2}{|c|}{ Adjusted c.s. values ${ }^{\mathrm{b}}$} & \multirow{2}{*}{\multicolumn{5}{|c|}{$\frac{\text { Chemical shift difference } \Delta \delta_{i}}{\left(2^{\prime} \text {-ethyl taxane }\right)-\left(2^{\prime} \text {-methyl taxane }\right)}$}} \\
\hline & & \multirow{2}{*}{$\begin{array}{l}\text { 2'-ethyl } \\
\text { taxane } \\
\text { minor } \\
\text { epimer } \\
\text { (3a) }\end{array}$} & \multirow{2}{*}{$\begin{array}{c}\text { 2'-ethyl } \\
\text { taxane } \\
\text { major } \\
\text { epimer } \\
(\mathbf{3 b})\end{array}$} & & & & & \\
\hline & & & & $3 a-s y n$ & 3a-anti & & $3 b-s y n$ & $3 b-a n t i$ \\
\hline $\begin{array}{l}\text { Carbon } \\
\text { number }\end{array}$ & ${ }^{13} \mathrm{C}$ NMR & ${ }^{13} \mathrm{C}$ NMR & ${ }^{13} \mathrm{C}$ NMR & ${ }^{13} \mathrm{C}$ NMR & ${ }^{13} \mathrm{C}$ NMR & & ${ }^{13} \mathrm{C} \mathrm{NMR}$ & ${ }^{13} \mathrm{C}$ NMR \\
\hline 1 & $-0,85$ & 177,19 & 176,52 & 0,29 & 0,39 & & $-0,38$ & $-0,28$ \\
\hline 2 & 3,75 & 78,01 & 78,20 & 0,00 & $-0,20$ & & 0,19 & $-0,01$ \\
\hline 3 & $-1,23$ & 55,30 & 55,50 & $-0,40$ & 0,20 & & $-0,20$ & 0,40 \\
\hline 4 & 0,50 & 38,51 & 39,50 & 0,31 & $-1,29$ & & 1,30 & $-0,30$ \\
\hline 5 & $-0,48$ & 25,30 & 25,61 & $-0,11$ & $-0,21$ & & 0,21 & 0,11 \\
\hline 6 & 0,33 & 21,54 & 21,36 & 0,04 & 0,14 & & $-0,14$ & $-0,04$ \\
\hline 7 & 0,63 & 23,83 & 23,58 & $-0,18$ & 0,43 & & $-0,43$ & 0,18 \\
\hline 8 & $-0,92$ & 156,53 & 157,28 & $-0,08$ & $-0,68$ & & 0,67 & 0,07 \\
\hline 9 & 0,40 & 79,20 & 79,20 & 0,00 & 0,00 & & 0,00 & 0,00 \\
\hline 10 & 0,06 & 28,32 & 28,59 & $-0,09$ & $-0,19$ & & 0,18 & 0,08 \\
\hline 11 & 5,65 & 22,89 & 23,92 & $-0,02$ & $-1,02$ & & 1,02 & 0,01 \\
\hline & & & & 1,49 & 4,72 & TAD $^{c}$ & 4,72 & 1,49 \\
\hline
\end{tabular}

a) correcting factor: $\mathrm{CF}_{\mathrm{i}}=\mathrm{m}_{\mathrm{i}}^{\text {epimers }}-\mathrm{m}_{\mathrm{i}}{ }^{\mathrm{DB}}$, where $\mathrm{m}_{\mathrm{i}}$ 's are mean chemical shift values

b) adjusted chemical shift values, obtained $c . s_{\cdot \mathrm{i}}=\mathrm{c} . \mathrm{s}_{\mathrm{i}}-\mathrm{CF}_{\mathrm{i}}$

c) $\mathrm{TAD}=$ Total Absolute Deviation: sum of absolute values of all c.s. differences $=\Sigma_{\mathrm{i}}\left|\Delta \delta_{\mathrm{i}}\right|$

Minor epimer (3a): $\quad$ Syn

Major epimer (3b): Anti 


\section{2'-Isopropyltaxanes:}

Table S12: Side chain ${ }^{13} \mathrm{C}$ NMR c.s. values of the epimeric 2 -methyl taxanes and the compounds under investigation

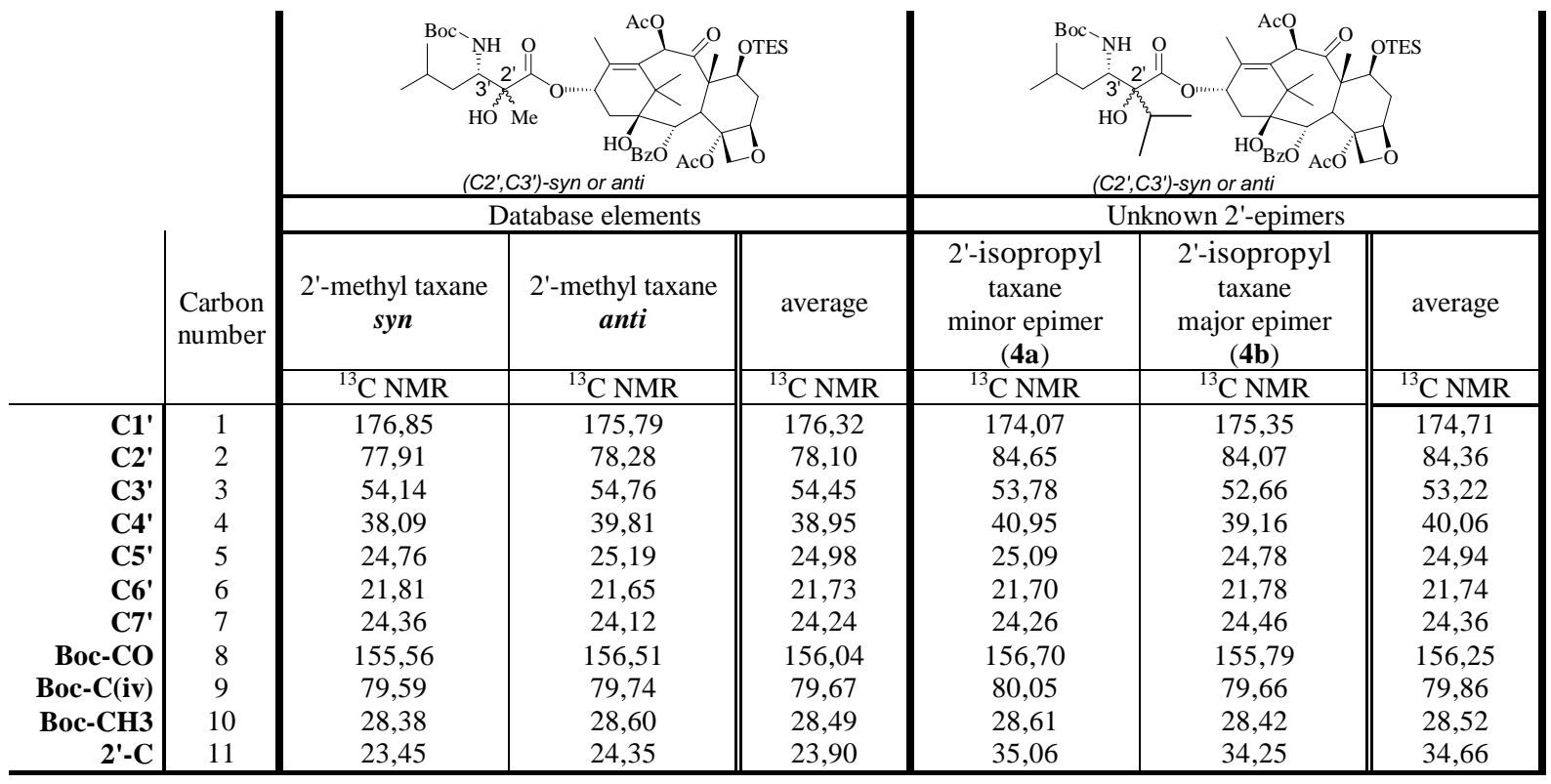

\section{Original UBD analysis}

Table S13: Original UDB analysis performed on title compound

\begin{tabular}{|c|c|c|c|c|c|c|c|c|c|c|}
\hline \multirow{4}{*}{$\begin{array}{l}\text { Carbon } \\
\text { number }\end{array}$} & \multicolumn{2}{|c|}{ Calculated c.s. ${ }^{\mathrm{a}}$} & \multirow[b]{3}{*}{$\begin{array}{l}\text { Correcting } \\
\text { factor }^{\mathrm{b}}\end{array}$} & \multicolumn{2}{|c|}{ Adjusted c.s. ${ }^{\mathrm{c}}$} & \multicolumn{5}{|c|}{ Chemical shift difference } \\
\hline & \multirow[b]{2}{*}{$\begin{array}{l}2 \text { 'methyl } \\
\text { taxane }\end{array}$} & \multirow[b]{2}{*}{$\begin{array}{c}2^{\prime} \\
\text { isopropyl } \\
\text { taxane }\end{array}$} & & \multirow{2}{*}{$\begin{array}{c}2^{\prime}- \\
\text { isopropyl } \\
\text { taxane } \\
\text { minor } \\
\text { epimer } \\
\text { (4a) } \\
\end{array}$} & \multirow{2}{*}{$\begin{array}{c}2 '- \\
\text { isopropyl } \\
\text { taxane } \\
\text { major } \\
\text { epimer } \\
\text { (4b) } \\
\end{array}$} & \multicolumn{5}{|c|}{ (2'-methyl taxane) - (adjusted 2'- isopropyl taxane) } \\
\hline & & & & & & syn - 4a & anti - 4a & & syn $-4 b$ & anti - 4b \\
\hline & $\begin{array}{c}{ }^{13} \mathrm{C} \\
\mathrm{NMR}\end{array}$ & $\begin{array}{c}{ }^{13} \mathrm{C} \\
\mathrm{NMR}\end{array}$ & ${ }^{13} \mathrm{C} \mathrm{NMR}$ & ${ }^{13} \mathrm{C}$ NMR & ${ }^{13} \mathrm{C} \mathrm{NMR}$ & ${ }^{13} \mathrm{C}$ NMR & ${ }^{13} \mathrm{C}$ NMR & & ${ }^{13} \mathrm{C}$ NMR & ${ }^{13} \mathrm{C} \mathrm{NMR}$ \\
\hline 1 & 174,50 & 176,00 & $-1,50$ & 172,57 & 173,85 & 4,28 & 3,22 & & 3,00 & 1,94 \\
\hline 2 & 87,90 & 98,20 & $-10,30$ & 74,35 & 73,77 & 3,56 & 3,93 & & 4,14 & 4,51 \\
\hline 3 & 55,30 & 50,30 & 5,00 & 58,78 & 57,66 & $-4,64$ & $-4,02$ & & $-3,52$ & $-2,90$ \\
\hline 4 & 36,10 & 36,70 & $-0,60$ & 40,35 & 38,56 & $-2,26$ & $-0,54$ & & $-0,47$ & 1,25 \\
\hline 5 & 23,70 & 23,70 & 0,00 & 25,09 & 24,78 & $-0,33$ & 0,10 & & $-0,02$ & 0,41 \\
\hline 6 & 22,30 & 22,30 & 0,00 & 21,70 & 21,78 & 0,11 & $-0,05$ & & 0,03 & $-0,13$ \\
\hline 7 & 22,30 & 22,30 & 0,00 & 24,26 & 24,46 & 0,10 & $-0,14$ & & $-0,10$ & $-0,34$ \\
\hline 8 & 157,20 & 157,20 & 0,00 & 156,70 & 155,79 & $-1,14$ & $-0,19$ & & $-0,23$ & 0,72 \\
\hline 9 & 70,60 & 70,60 & 0,00 & 80,05 & 79,66 & $-0,46$ & $-0,31$ & & $-0,07$ & 0,08 \\
\hline 10 & 28,70 & 28,70 & 0,00 & 28,61 & 28,42 & $-0,23$ & $-0,01$ & & $-0,04$ & 0,18 \\
\hline 11 & 17,90 & 29,50 & $-11,60$ & 23,46 & 22,65 & $-0,01$ & 0,89 & & 0,80 & 1,70 \\
\hline & & & & & & 17,12 & 13,40 & $\mathbf{T A D}^{\mathrm{d}}$ & 12,42 & 14,16 \\
\hline
\end{tabular}

a) CS ChemNMR Pro version 1.0, Renate Buergin Schaller, Development Centre, Bergstr. 114, Zurich, Switzerland, installed in CS ChemDraw Pro version 7.0, was used.

b) Correcting factor $=($ database elements c.s. calculated value $)-($ unknown 2'-epimers c.s. calculated value $)$

c) adjusted c.s. value $=($ unknown 2 '-epimer c.s. measured value $)+($ correcting factor $)$

d) $\mathrm{TAD}=$ Total Absolute Deviation: sum of absolute values of all c.s. differences $=\Sigma_{\mathrm{i}}\left|\Delta \delta_{\mathrm{i}}\right|$ 


\section{Modified UDB approach}

Table S14: Modified UDB analysis performed on title compound.

\begin{tabular}{|c|c|c|c|c|c|c|c|c|}
\hline \multirow{4}{*}{$\begin{array}{l}\text { Carbon } \\
\text { number }\end{array}$} & \multirow[b]{3}{*}{$\begin{array}{c}\text { Correcting } \\
\text { factor }^{\mathrm{a}}\end{array}$} & \multicolumn{2}{|c|}{ Adjusted c.s. values ${ }^{\mathrm{b}}$} & \multicolumn{5}{|c|}{ Chemical shift difference $\Delta \delta_{i}$} \\
\hline & & \multirow[b]{2}{*}{$\begin{array}{c}2 '- \\
\text { isopropyl } \\
\text { taxane } \\
\text { minor } \\
\text { epimer } \\
(\mathbf{4 a})\end{array}$} & \multirow[b]{2}{*}{$\begin{array}{c}2 '- \\
\text { isopropyl } \\
\text { taxane } \\
\text { major } \\
\text { epimer } \\
\text { (4b) }\end{array}$} & \multicolumn{5}{|c|}{ (2'-isopropyl taxane) - (2'-methyl taxane) } \\
\hline & & & & $4 a-s y n$ & $4 a-a n t i$ & & $4 b-s y n$ & $4 \mathrm{~b}-$ anti \\
\hline & ${ }^{13} \mathrm{C}$ NMR & ${ }^{13} \mathrm{C}$ NMR & ${ }^{13} \mathrm{C}$ NMR & ${ }^{13} \mathrm{C}$ NMR & ${ }^{13} \mathrm{C} \mathrm{NMR}$ & & ${ }^{13} \mathrm{C} \mathrm{NMR}$ & ${ }^{13} \mathrm{C} \mathrm{NMR}$ \\
\hline 1 & $-1,61$ & 175,68 & 176,96 & $-1,17$ & $-0,11$ & & 0,11 & 1,17 \\
\hline 2 & 6,27 & 78,39 & 77,81 & 0,48 & 0,11 & & $-0,11$ & $-0,48$ \\
\hline 3 & $-1,23$ & 55,01 & 53,89 & 0,87 & 0,25 & & $-0,25$ & $-0,87$ \\
\hline 4 & 1,11 & 39,85 & 38,06 & 1,76 & 0,04 & & $-0,04$ & $-1,76$ \\
\hline 5 & $-0,04$ & 25,13 & 24,82 & 0,37 & $-0,06$ & & 0,06 & $-0,37$ \\
\hline 6 & 0,01 & 21,69 & 21,77 & $-0,12$ & 0,04 & & $-0,04$ & 0,12 \\
\hline 7 & 0,12 & 24,14 & 24,34 & $-0,22$ & 0,02 & & $-0,02$ & 0,22 \\
\hline 8 & 0,21 & 156,49 & 155,58 & 0,93 & -0.02 & & 0,02 & $-0,93$ \\
\hline 9 & 0,19 & 79,86 & 79,47 & 0,27 & 0,12 & & $-0,12$ & $-0,27$ \\
\hline 10 & 0,02 & 28,59 & 28,40 & 0,21 & $-0,02$ & & 0,02 & $-0,20$ \\
\hline 11 & 10,76 & 24,31 & 23,50 & 0,86 & $-0,05$ & & 0,04 & $-0,86$ \\
\hline & & & & 7,24 & $\mathbf{0 , 8 2}$ & TAD $^{c}$ & $\mathbf{0 , 8 2}$ & 7,24 \\
\hline
\end{tabular}

a) correcting factor: $\mathrm{CF}_{\mathrm{i}}=\mathrm{m}_{\mathrm{i}}{ }^{\text {epimers }}-\mathrm{m}_{\mathrm{i}}{ }^{\mathrm{DB}}$, where $\mathrm{m}_{\mathrm{i}}$ 's are mean chemical shift values

b) adjusted chemical shift values, obtained $c . s ._{\mathrm{i}}=\mathrm{c} . \mathrm{s}_{\mathrm{i}_{\mathrm{i}}}-\mathrm{CF}_{\mathrm{i}}$

c) $\mathrm{TAD}=$ Total Absolute Deviation: sum of absolute values of all c.s. differences $=\Sigma_{\mathrm{i}}\left|\Delta \delta_{\mathrm{i}}\right|$

Minor epimer (4a): $\mid$ Anti

Major epimer (4b): $\quad$ Syn 


\section{2'-tert-Butyltaxanes:}

A) Comparison of the major conformers of the diastereomeric 2'-tertbutyl taxanes with 2'-methyl taxanes

Table S15: Side chain ${ }^{13} \mathrm{C}$ NMR c.s. values of the epimeric 2'-methyl taxanes and the compounds under investigation

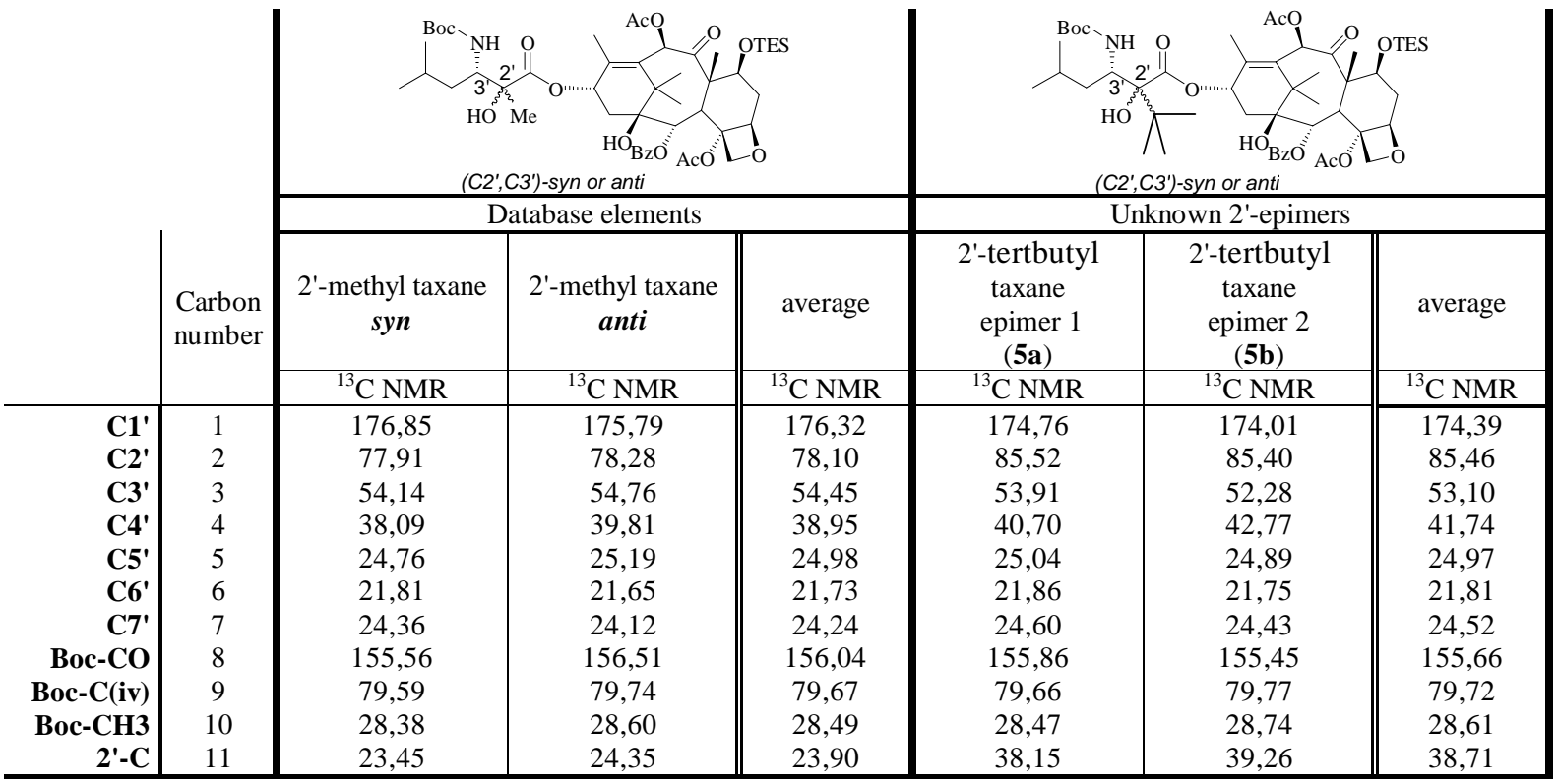

\section{Original UBD analysis}

Table S16: Original UDB analysis performed on title compound

\begin{tabular}{|c|c|c|c|c|c|c|c|c|c|c|}
\hline \multirow{4}{*}{$\begin{array}{l}\text { Carbon } \\
\text { number }\end{array}$} & \multicolumn{2}{|c|}{ Calculated c.s. ${ }^{\mathrm{a}}$} & \multirow[b]{3}{*}{$\begin{array}{l}\text { Correcting } \\
\text { factor }^{b}\end{array}$} & \multicolumn{2}{|c|}{ Adjusted c.s. ${ }^{c}$} & \multicolumn{5}{|c|}{ Chemical shift difference } \\
\hline & \multirow[b]{2}{*}{$\begin{array}{l}\text { 2'methyl } \\
\text { taxane }\end{array}$} & \multirow[b]{2}{*}{$\begin{array}{c}2^{\prime}- \\
\text { tertbutyl } \\
\text { taxane }\end{array}$} & & \multirow{2}{*}{$\begin{array}{c}2^{\prime}- \\
\text { tertbutyl } \\
\text { taxane } \\
\text { epimer } 1 \\
(\mathbf{5 a})\end{array}$} & \multirow{2}{*}{$\begin{array}{c}2^{\prime}- \\
\text { tertbutyl } \\
\text { taxane } \\
\text { epimer } 2 \\
(\mathbf{5 b})\end{array}$} & \multicolumn{5}{|c|}{ (2'-methyl taxane) - (adjusted 2'-tertbutyl taxane) } \\
\hline & & & & & & syn - 5a & anti - 5a & & syn - 5b & anti - 5b \\
\hline & $\begin{array}{c}{ }^{13} \mathrm{C} \\
\mathrm{NMR}\end{array}$ & $\begin{array}{c}{ }^{13} \mathrm{C} \\
\mathrm{NMR}\end{array}$ & ${ }^{13} \mathrm{C}$ NMR & ${ }^{13} \mathrm{C}$ NMR & ${ }^{13} \mathrm{C}$ NMR & ${ }^{13} \mathrm{C}$ NMR & ${ }^{13} \mathrm{C}$ NMR & & ${ }^{13} \mathrm{C}$ NMR & ${ }^{13} \mathrm{C}$ NMR \\
\hline 1 & 174,50 & 176,00 & 1,50 & 173,26 & 172,51 & $-3,59$ & $-2,53$ & & $-4,34$ & $-3,28$ \\
\hline 2 & 87,90 & 105,10 & 17,20 & 68,32 & 68,20 & $-9,59$ & $-9,96$ & & $-9,71$ & $-10,08$ \\
\hline 3 & 55,30 & 47,80 & $-7,50$ & 61,41 & 59,78 & 7,27 & 6,65 & & 5,64 & 5,02 \\
\hline 4 & 36,10 & 37,00 & 0,90 & 39,80 & 41,87 & 1,71 & $-0,01$ & & 3,78 & 2,06 \\
\hline 5 & 23,70 & 23,70 & 0,00 & 25,04 & 24,89 & 0,28 & $-0,15$ & & 0,13 & $-0,30$ \\
\hline 6 & 22,30 & 22,30 & 0,00 & 21,86 & 21,75 & 0,05 & 0,21 & & $-0,06$ & 0,10 \\
\hline 7 & 22,30 & 22,30 & 0,00 & 24,60 & 24,43 & 0,24 & 0,48 & & 0,07 & 0,31 \\
\hline 8 & 157,20 & 157,20 & 0,00 & 155,86 & 155,45 & 0,30 & $-0,65$ & & $-0,11$ & $-1,06$ \\
\hline 9 & 70,60 & 70,60 & 0,00 & 79,66 & 79,77 & 0,07 & $-0,08$ & & 0,18 & 0,03 \\
\hline 10 & 28,70 & 28,70 & 0,00 & 28,47 & 28,74 & 0,09 & $-0,13$ & & 0,36 & 0,14 \\
\hline 11 & 17,90 & 31,60 & 13,70 & 24,45 & 25,56 & 1,00 & 0,10 & & 2,11 & 1,21 \\
\hline & & & & & & 24,19 & 20,95 & $\begin{array}{c}\mathbf{T} \\
\mathbf{A} \\
\mathbf{D}^{\mathrm{d}}\end{array}$ & 26,49 & 23,59 \\
\hline
\end{tabular}

a) CS ChemNMR Pro version 1.0, Renate Buergin Schaller, Development Centre, Bergstr. 114, Zurich, Switzerland, installed in CS ChemDraw Pro version 7.0, was used.

b) Correcting factor $=($ database elements c.s. calculated value $)-($ unknown 2 -epimers c.s. calculated value $)$

c) adjusted c.s. value $=($ unknown 2 '-epimer c.s. measured value $)+($ correcting factor $)$

d) $\mathrm{TAD}=$ Total Absolute Deviation: sum of absolute values of all c.s. differences $=\Sigma_{\mathrm{i}}\left|\Delta \delta_{\mathrm{i}}\right|$ 


\section{Modified UDB approach}

Table S17: Modified UDB analysis performed on title compound.

\begin{tabular}{|c|c|c|c|c|c|c|c|c|}
\hline \multirow{4}{*}{$\begin{array}{l}\text { Carbon } \\
\text { number }\end{array}$} & \multirow[b]{3}{*}{$\begin{array}{c}\text { Correcting } \\
\text { factor }^{\mathrm{a}}\end{array}$} & \multicolumn{2}{|c|}{ Adjusted c.s. values $^{\mathrm{b}}$} & \multicolumn{5}{|c|}{ Chemical shift difference $\Delta \delta_{i}$} \\
\hline & & \multirow{2}{*}{$\begin{array}{c}2 '- \\
\text { tertbutyl } \\
\text { taxane } \\
\text { epimer } 1 \\
(\mathbf{5 a})\end{array}$} & \multirow{2}{*}{$\begin{array}{c}2 '- \\
\text { tertbutyl } \\
\text { taxane } \\
\text { epimer } 2 \\
(\mathbf{5 b})\end{array}$} & \multicolumn{5}{|c|}{ (2'-tertbutyl taxane) - (2'-methyl taxane) } \\
\hline & & & & $5 \mathbf{a}-s y n$ & 5a-anti & & $5 \mathbf{b}-s y n$ & $5 \mathbf{b}-$ anti \\
\hline & ${ }^{13} \mathrm{C}$ NMR & ${ }^{13} \mathrm{C}$ NMR & ${ }^{13} \mathrm{C}$ NMR & ${ }^{13} \mathrm{C}$ NMR & ${ }^{13} \mathrm{C}$ NMR & & ${ }^{13} \mathrm{C} \mathrm{NMR}$ & ${ }^{13} \mathrm{C}$ NMR \\
\hline 1 & $-1,94$ & 176,70 & 175,95 & $-0,16$ & 0,91 & & $-0,91$ & 0,16 \\
\hline 2 & 7,37 & 78,16 & 78,04 & 0,24 & $-0,13$ & & 0,13 & $-0,25$ \\
\hline 3 & $-1,36$ & 55,27 & 53,64 & 1,13 & 0,51 & & $-0,50$ & $-1,12$ \\
\hline 4 & 2,79 & 37,92 & 39,99 & $-0,17$ & $-1,90$ & & 1,90 & 0,18 \\
\hline 5 & $-0,01$ & 25,05 & 24,90 & 0,29 & $-0,14$ & & 0,14 & $-0,29$ \\
\hline 6 & 0,08 & 21,79 & 21,68 & $-0,03$ & 0,13 & & $-0,14$ & 0,02 \\
\hline 7 & 0,27 & 24,33 & 24,16 & $-0,03$ & 0,21 & & $-0,20$ & 0,04 \\
\hline 8 & $-0,38$ & 156,24 & 155,83 & 0,68 & $-0,27$ & & 0,27 & $-0,68$ \\
\hline 9 & 0,05 & 79,61 & 79,72 & 0,02 & $-0,13$ & & 0,13 & $-0,02$ \\
\hline 10 & 0,11 & 28,36 & 28,63 & $-0,02$ & $-0,24$ & & 0,25 & 0,03 \\
\hline 11 & 14,81 & 23,35 & 24,46 & $-0,11$ & $-1,01$ & & 1,01 & 0,10 \\
\hline & & & & 2,88 & 5,58 & TAD $^{c}$ & 5,58 & $\mathbf{2 , 8 8}$ \\
\hline
\end{tabular}

a) correcting factor: $\mathrm{CF}_{\mathrm{i}}=\mathrm{m}_{\mathrm{i}}^{\text {epimers }}-\mathrm{m}_{\mathrm{i}}{ }^{\mathrm{DB}}$, where $\mathrm{m}_{\mathrm{i}}$ 's are mean chemical shift values

b) adjusted chemical shift values, obtained $c . s_{\cdot \mathrm{i}}=\mathrm{C} . \mathrm{s}_{\cdot \mathrm{i}}-\mathrm{CF}_{\mathrm{i}}$

c) $\mathrm{TAD}=$ Total Absolute Deviation: sum of absolute values of all c.s. differences $=\Sigma_{\mathrm{i}}\left|\Delta \delta_{\mathrm{i}}\right|$

Epimer 1(5a): $\quad$ Syn

Epimer 2(5b): $\quad$ Anti 
B) Comparison of the minor conformers of the diastereomeric 2'-tertbutyl taxanes with 2'-methyl taxanes

Table S18: Side chain ${ }^{13} \mathrm{C}$ NMR c.s. values of the epimeric 2 -methyl taxanes and the compounds under investigation

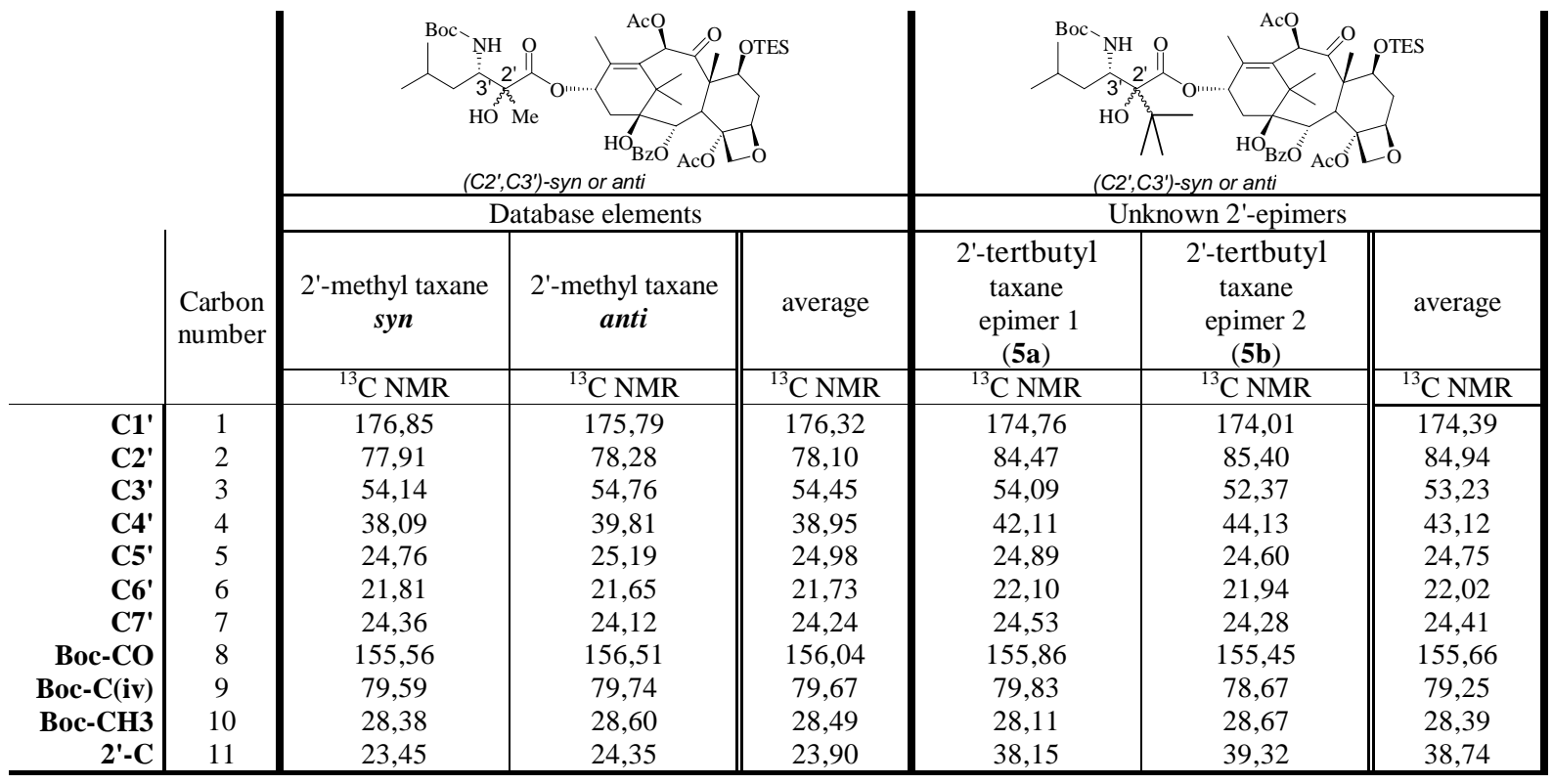

\section{Original UBD analysis}

Table S19: Original UDB analysis performed on title compound

\begin{tabular}{|c|c|c|c|c|c|c|c|c|c|c|}
\hline \multirow{4}{*}{$\begin{array}{l}\text { Carbon } \\
\text { number }\end{array}$} & \multicolumn{2}{|c|}{ Calculated c.s. $^{\mathrm{a}}$} & \multirow{3}{*}{$\begin{array}{l}\text { Correcting } \\
\text { factor }^{\mathrm{b}}\end{array}$} & \multicolumn{2}{|c|}{ Adjusted c.s. ${ }^{\mathrm{c}}$} & \multicolumn{5}{|c|}{ Chemical shift difference } \\
\hline & \multirow[b]{2}{*}{$\begin{array}{l}2 \text { 'methyl } \\
\text { taxane }\end{array}$} & \multirow[b]{2}{*}{$\begin{array}{c}2^{\prime}- \\
\text { tertbutyl } \\
\text { taxane }\end{array}$} & & \multirow{2}{*}{$\begin{array}{c}2^{\prime}- \\
\text { tertbutyl } \\
\text { taxane } \\
\text { epimer } 1 \\
\text { (5a) }\end{array}$} & \multirow{2}{*}{$\begin{array}{c}2 '- \\
\text { tertbutyl } \\
\text { taxane } \\
\text { epimer } 2 \\
(\mathbf{5 b})\end{array}$} & \multicolumn{5}{|c|}{ (2'-methyl taxane) - (adjusted 2'-tertbutyl taxane) } \\
\hline & & & & & & syn $-\mathbf{5 a}$ & anti - 5a & & syn $-\mathbf{5 b}$ & anti $-\mathbf{5 b}$ \\
\hline & $\begin{array}{c}{ }^{13} \mathrm{C} \\
\mathrm{NMR}\end{array}$ & $\begin{array}{c}{ }^{13} \mathrm{C} \\
\mathrm{NMR}\end{array}$ & ${ }^{13} \mathrm{C}$ NMR & ${ }^{13} \mathrm{C}$ NMR & ${ }^{13} \mathrm{C}$ NMR & ${ }^{13} \mathrm{C}$ NMR & ${ }^{13} \mathrm{C} \mathrm{NMR}$ & & ${ }^{13} \mathrm{C}$ NMR & ${ }^{13} \mathrm{C}$ NMR \\
\hline 1 & 174,50 & 176,00 & 1,50 & 173,26 & 172,51 & $-3,59$ & $-2,53$ & & $-4,34$ & $-3,28$ \\
\hline 2 & 87,90 & 105,10 & 17,20 & 67,27 & 68,20 & $-10,64$ & $-11,01$ & & $-9,71$ & $-10,08$ \\
\hline 3 & 55,30 & 47,80 & $-7,50$ & 61,59 & 59,87 & 7,45 & 6,83 & & 5,73 & 5,11 \\
\hline 4 & 36,10 & 37,00 & 0,90 & 41,21 & 43,23 & 3,12 & 1,40 & & 5,14 & 3,42 \\
\hline 5 & 23,70 & 23,70 & 0,00 & 24,89 & 24,60 & 0,13 & $-0,30$ & & $-0,16$ & $-0,59$ \\
\hline 6 & 22,30 & 22,30 & 0,00 & 22,10 & 21,94 & 0,29 & 0,45 & & 0,13 & 0,29 \\
\hline 7 & 22,30 & 22,30 & 0,00 & 24,53 & 24,28 & 0,17 & 0,41 & & $-0,08$ & 0,16 \\
\hline 8 & 157,20 & 157,20 & 0,00 & 155,86 & 155,45 & 0,30 & $-0,65$ & & $-0,11$ & $-1,06$ \\
\hline 9 & 70,60 & 70,60 & 0,00 & 79,83 & 78,67 & 0,24 & 0,09 & & $-0,92$ & $-1,07$ \\
\hline 10 & 28,70 & 28,70 & 0,00 & 28,11 & 28,67 & $-0,27$ & $-0,49$ & & 0,29 & 0,07 \\
\hline 11 & 17,90 & 31,60 & 13,70 & 24,45 & 25,62 & 1,00 & 0,10 & & 2,17 & 1,27 \\
\hline & & & & & & 27,20 & 24,26 & $\begin{array}{c}\mathbf{T} \\
\mathbf{A} \\
\mathbf{D}^{\mathrm{d}}\end{array}$ & 28,78 & 26,40 \\
\hline
\end{tabular}

a) CS ChemNMR Pro version 1.0, Renate Buergin Schaller, Development Centre, Bergstr. 114, Zurich, Switzerland, installed in CS ChemDraw Pro version 7.0 , was used.

b) Correcting factor $=($ database elements c.s. calculated value $)-($ unknown 2 '-epimers c.s. calculated value $)$

c) adjusted c.s. value $=($ unknown 2 '-epimer c.s. measured value $)+($ correcting factor $)$

d) $\mathrm{TAD}=$ Total Absolute Deviation: sum of absolute values of all c.s. differences $=\Sigma_{\mathrm{i}}\left|\Delta \delta_{\mathrm{i}}\right|$ 


\section{Modified UDB approach}

Table S20: $\quad$ Modified UDB analysis performed on title compound.

\begin{tabular}{|c|c|c|c|c|c|c|c|c|}
\hline \multirow{4}{*}{$\begin{array}{l}\text { Carbon } \\
\text { number }\end{array}$} & \multirow[b]{3}{*}{$\begin{array}{c}\text { Correcting } \\
\text { factor }^{\mathrm{a}}\end{array}$} & \multicolumn{2}{|c|}{ Adjusted c.s. values ${ }^{b}$} & \multicolumn{5}{|c|}{ Chemical shift difference $\Delta \delta_{i}$} \\
\hline & & & & \multicolumn{5}{|c|}{ (2'-tertbutyl taxane) - (2'-methyl taxane) } \\
\hline & & $\begin{array}{c}\text { tertbutyl } \\
\text { taxane } \\
\text { epimer } 1 \\
(\mathbf{5 a})\end{array}$ & $\begin{array}{c}\text { tertbutyl } \\
\text { taxane } \\
\text { epimer } 2 \\
(\mathbf{5 b})\end{array}$ & $5 \mathbf{a}-s y n$ & $5 \mathbf{a}-a n t i$ & & $5 b-s y n$ & $5 b-a n t i$ \\
\hline & ${ }^{13} \mathrm{C}$ NMR & ${ }^{13} \mathrm{C}$ NMR & ${ }^{13} \mathrm{C}$ NMR & ${ }^{13} \mathrm{C}$ NMR & ${ }^{13} \mathrm{C} \mathrm{NMR}$ & & ${ }^{13} \mathrm{C} \mathrm{NMR}$ & ${ }^{13} \mathrm{C} \mathrm{NMR}$ \\
\hline 1 & $-1,94$ & 176,70 & 175,95 & $-0,16$ & 0,91 & & $-0,91$ & 0,16 \\
\hline 2 & 6,84 & 77,63 & 78,56 & $-0,28$ & $-0,65$ & & 0,65 & 0,28 \\
\hline 3 & $-1,22$ & 55,31 & 53,59 & 1,17 & 0,55 & & $-0,55$ & $-1,17$ \\
\hline 4 & 4,17 & 37,94 & 39,96 & $-0,15$ & $-1,87$ & & 1,87 & 0,15 \\
\hline 5 & $-0,23$ & 25,12 & 24,83 & 0,36 & $-0,07$ & & 0,07 & $-0,36$ \\
\hline 6 & 0,29 & 21,81 & 21,65 & 0,00 & 0,16 & & $-0,16$ & 0,00 \\
\hline 7 & 0,16 & 24,37 & 24,12 & 0,01 & 0,25 & & $-0,24$ & 0,00 \\
\hline 8 & $-0,38$ & 156,24 & 155,83 & 0,68 & $-0,27$ & & 0,27 & $-0,68$ \\
\hline 9 & $-0,41$ & 80,25 & 79,09 & 0,65 & 0,50 & & $-0,51$ & $-0,66$ \\
\hline 10 & $-0,10$ & 28,21 & 28,77 & $-0,17$ & $-0,39$ & & 0,39 & 0,17 \\
\hline 11 & 14,84 & 23,32 & 24,49 & $-0,14$ & $-1,04$ & & 1,04 & 0,13 \\
\hline & & & & 3,76 & 6,65 & $\mathbf{T A D}^{\mathrm{c}}$ & 6,65 & 3,76 \\
\hline
\end{tabular}

a) correcting factor: $\mathrm{CF}_{\mathrm{i}}=\mathrm{m}_{\mathrm{i}}^{\text {epimers }}-\mathrm{m}_{\mathrm{i}}^{\mathrm{DB}}$, where $\mathrm{m}_{\mathrm{i}}$ 's are mean chemical shift values

b) adjusted chemical shift values, obtained $\boldsymbol{C} . s_{\cdot \mathrm{i}}=\mathrm{C} . \mathrm{S}_{\cdot \mathrm{i}}-\mathrm{CF}_{\mathrm{i}}$

c) $\mathrm{TAD}=$ Total Absolute Deviation: sum of absolute values of all c.s. differences $=\Sigma_{\mathrm{i}}\left|\Delta \delta_{\mathrm{i}}\right|$

Epimer 1 (5a): $\quad$ Syn

Epimer 2 (5b): $\quad$ Anti

Figure S1: Histograms of modified UDB analysis for minor conformers of 2'-tertbutyl taxanes
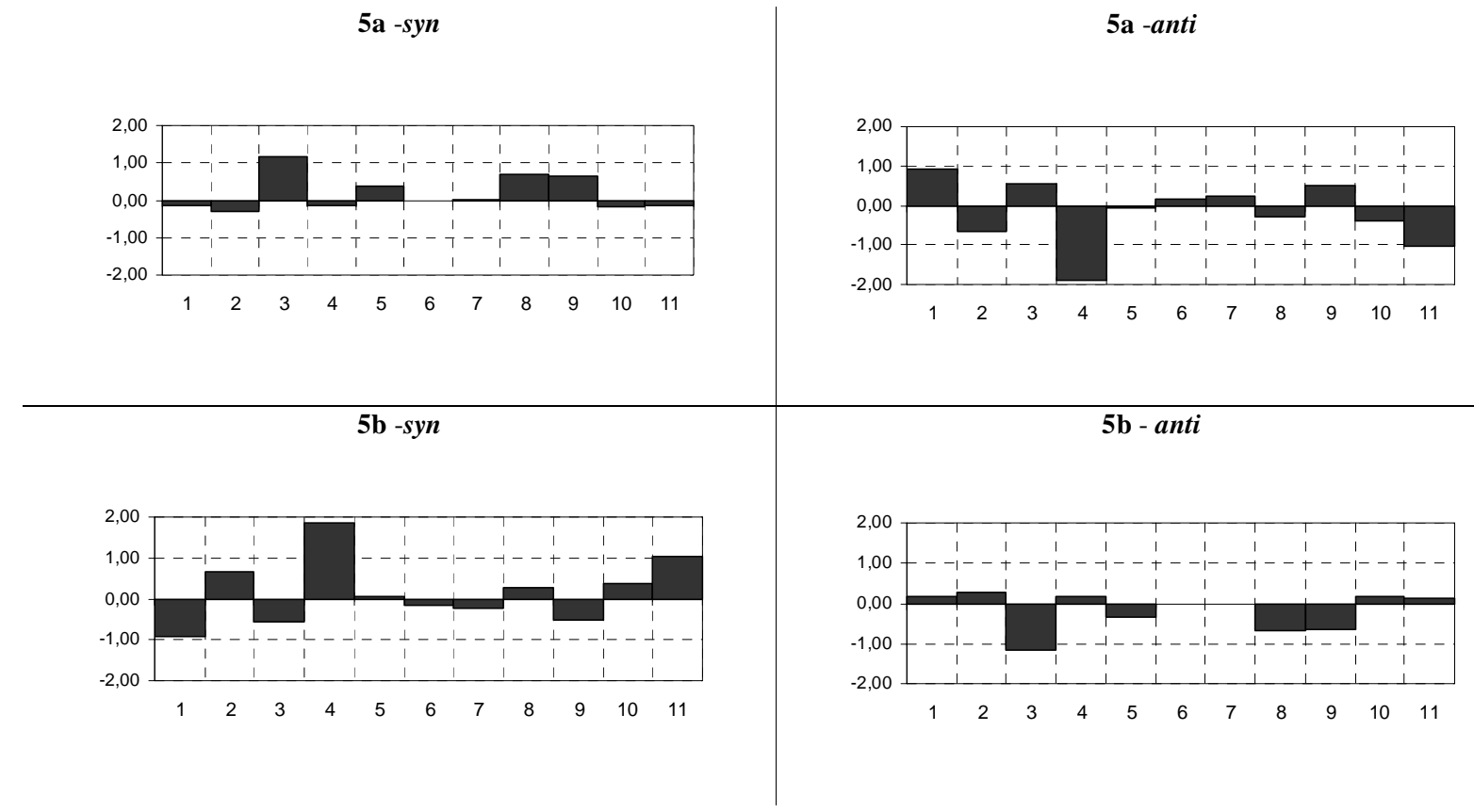
C) Comparison of the major conformer of epimer 1 of 2'-tertbutyl taxanes with the minor conformer of its diastereomer

Table S21: Side chain ${ }^{13} \mathrm{C}$ NMR c.s. values of the epimeric 2 -methyl taxanes and the compounds under investigation

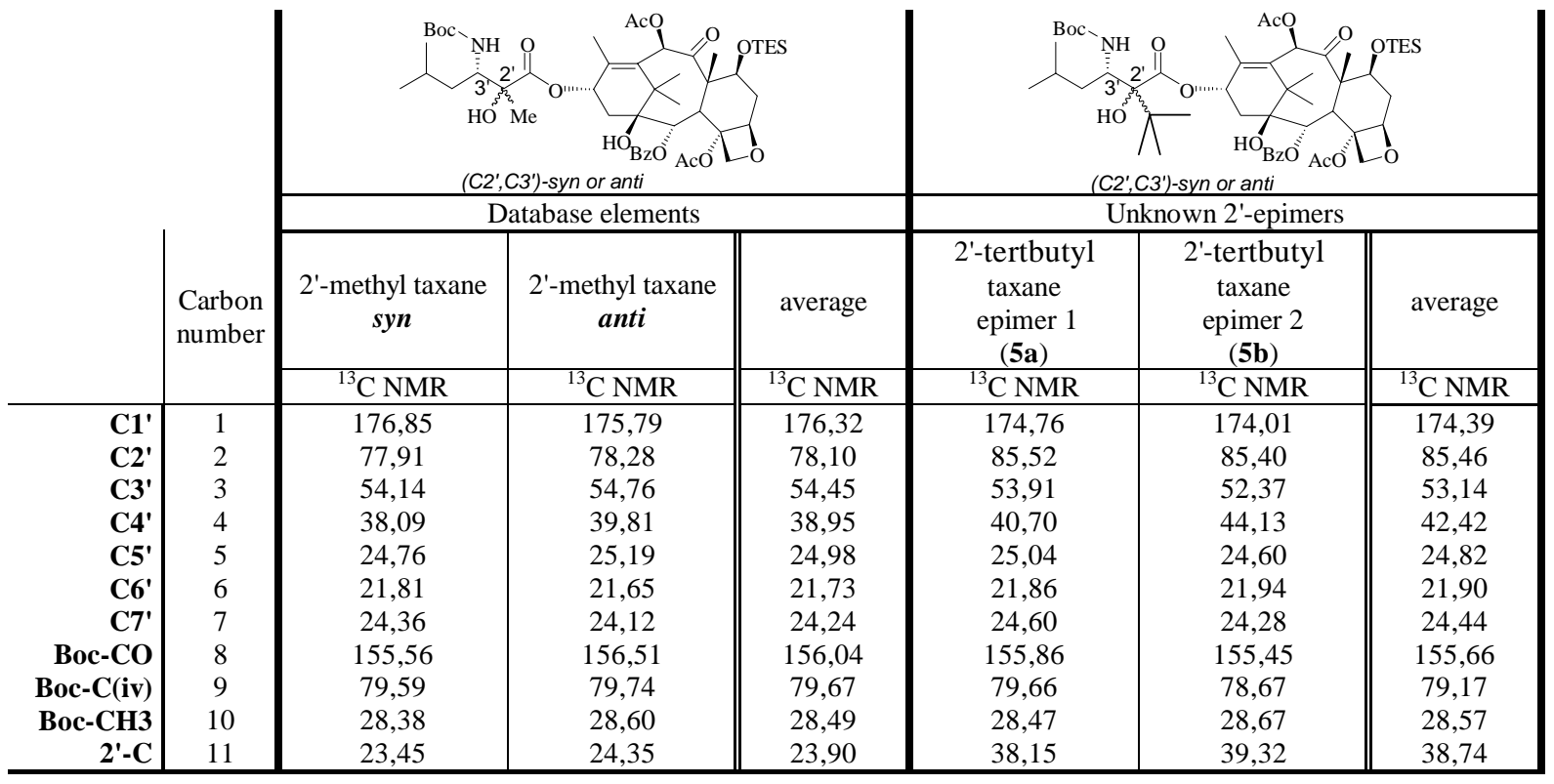

\section{Original UBD analysis}

Table S22: Original UDB analysis performed on title compound

\begin{tabular}{|c|c|c|c|c|c|c|c|c|c|c|}
\hline \multirow{4}{*}{$\begin{array}{l}\text { Carbon } \\
\text { number }\end{array}$} & \multicolumn{2}{|c|}{ Calculated c.s. ${ }^{\mathrm{a}}$} & \multirow{3}{*}{$\begin{array}{l}\text { Correcting } \\
\text { factor }^{\mathrm{b}}\end{array}$} & \multicolumn{2}{|c|}{ Adjusted c.s. ${ }^{c}$} & \multicolumn{5}{|c|}{ Chemical shift difference } \\
\hline & \multirow[b]{2}{*}{$\begin{array}{l}2 \text { 'methyl } \\
\text { taxane }\end{array}$} & \multirow[b]{2}{*}{$\begin{array}{l}2^{\prime}- \\
\text { tertbutyl } \\
\text { taxane }\end{array}$} & & \multirow{2}{*}{$\begin{array}{c}2^{\prime}- \\
\text { tertbutyl } \\
\text { taxane } \\
\text { epimer } 1 \\
\text { (5a) }\end{array}$} & \multirow{2}{*}{$\begin{array}{c}2^{\prime}- \\
\text { tertbutyl } \\
\text { taxane } \\
\text { epimer } 2 \\
(\mathbf{5 b})\end{array}$} & \multicolumn{5}{|c|}{ (2'-methyl taxane) - (adjusted 2'-tertbutyl taxane) } \\
\hline & & & & & & syn $-\mathbf{5 a}$ & anti - 5a & & syn $-\mathbf{5 b}$ & anti - $\mathbf{5 b}$ \\
\hline & $\begin{array}{c}{ }^{13} \mathrm{C} \\
\mathrm{NMR}\end{array}$ & $\begin{array}{c}{ }^{13} \mathrm{C} \\
\mathrm{NMR}\end{array}$ & ${ }^{13} \mathrm{C}$ NMR & ${ }^{13} \mathrm{C}$ NMR & ${ }^{13} \mathrm{C}$ NMR & ${ }^{13} \mathrm{C}$ NMR & ${ }^{13} \mathrm{C}$ NMR & & ${ }^{13} \mathrm{C} \mathrm{NMR}$ & ${ }^{13} \mathrm{C}$ NMR \\
\hline 1 & 174,50 & 176,00 & 1,50 & 173,26 & 172,51 & $-3,59$ & $-2,53$ & & $-4,34$ & $-3,28$ \\
\hline 2 & 87,90 & 105,10 & 17,20 & 68,32 & 68,20 & $-9,59$ & $-9,96$ & & $-9,71$ & $-10,08$ \\
\hline 3 & 55,30 & 47,80 & $-7,50$ & 61,41 & 59,87 & 7,27 & 6,65 & & 5,73 & 5,11 \\
\hline 4 & 36,10 & 37,00 & 0,90 & 39,80 & 43,23 & 1,71 & $-0,01$ & & 5,14 & 3,42 \\
\hline 5 & 23,70 & 23,70 & 0,00 & 25,04 & 24,60 & 0,28 & $-0,15$ & & $-0,16$ & $-0,59$ \\
\hline 6 & 22,30 & 22,30 & 0,00 & 21,86 & 21,94 & 0,05 & 0,21 & & 0,13 & 0,29 \\
\hline 7 & 22,30 & 22,30 & 0,00 & 24,60 & 24,28 & 0,24 & 0,48 & & $-0,08$ & 0,16 \\
\hline 8 & 157,20 & 157,20 & 0,00 & 155,86 & 155,45 & 0,30 & $-0,65$ & & $-0,11$ & $-1,06$ \\
\hline 9 & 70,60 & 70,60 & 0,00 & 79,66 & 78,67 & 0,07 & $-0,08$ & & $-0,92$ & $-1,07$ \\
\hline 10 & 28,70 & 28,70 & 0,00 & 28,47 & 28,67 & 0,09 & $-0,13$ & & 0,29 & 0,07 \\
\hline 11 & 17,90 & 31,60 & 13,70 & 24,45 & 25,62 & 1,00 & 0,10 & & 2,17 & 1,27 \\
\hline & & & & & & 24,19 & 20,95 & $\begin{array}{c}\mathbf{T} \\
\mathbf{A} \\
\mathbf{D}^{\mathrm{d}}\end{array}$ & 28,78 & 26,40 \\
\hline
\end{tabular}

a) CS ChemNMR Pro version 1.0, Renate Buergin Schaller, Development Centre, Bergstr. 114, Zurich, Switzerland, installed in CS ChemDraw Pro version 7.0, was used.

b) Correcting factor $=($ database elements c.s. calculated value $)-($ unknown 2'-epimers c.s. calculated value $)$

c) adjusted c.s. value $=($ unknown 2 '-epimer c.s. measured value $)+($ correcting factor $)$

d) $\mathrm{TAD}=$ Total Absolute Deviation: sum of absolute values of all c.s. differences $=\Sigma_{\mathrm{i}}\left|\Delta \delta_{\mathrm{i}}\right|$ 


\section{Modified UDB approach}

Table S23: Modified UDB analysis performed on title compound.

\begin{tabular}{|c|c|c|c|c|c|c|c|c|}
\hline \multirow{4}{*}{$\begin{array}{l}\text { Carbon } \\
\text { number }\end{array}$} & \multirow{3}{*}{$\begin{array}{l}\text { Correcting } \\
\text { factor }^{\mathrm{a}}\end{array}$} & \multicolumn{2}{|c|}{ Adjusted c.s. values ${ }^{\mathrm{b}}$} & \multirow{2}{*}{\multicolumn{5}{|c|}{$\frac{\text { Chemical shift difference } \Delta \delta_{\mathrm{i}}}{\text { (2'-tertbutyl taxane) }-\left(2^{2} \text {-methyl taxane }\right)}$}} \\
\hline & & \multirow{2}{*}{$\begin{array}{c}2 '- \\
\text { tertbutyl } \\
\text { taxane } \\
\text { epimer 1 } \\
(\mathbf{5 a}) \\
\end{array}$} & \multirow{2}{*}{$\begin{array}{c}2^{\prime}- \\
\text { tertbutyl } \\
\text { taxane } \\
\text { epimer } 2 \\
(\mathbf{5 b})\end{array}$} & & & & & \\
\hline & & & & $5 a-s y n$ & $5 \mathbf{a}-$ anti & & $5 b-s y n$ & $5 \mathbf{b}-a n t i$ \\
\hline & \begin{tabular}{|l|}
${ }^{13} \mathrm{C}$ NMR \\
\end{tabular} & ${ }^{13} \mathrm{C} N M R$ & ${ }^{13} \mathrm{C}$ NMR & ${ }^{13} \mathrm{C}$ NMR & ${ }^{13} \mathrm{C} \mathrm{NMR}$ & & ${ }^{13} \mathrm{C}$ NMR & ${ }^{13} \mathrm{C} \mathrm{NMR}$ \\
\hline 1 & $-1,94$ & 176,70 & 175,95 & $-0,16$ & 0,91 & & $-0,91$ & 0,16 \\
\hline 2 & 7,37 & 78,16 & 78,04 & 0,24 & $-0,13$ & & 0,13 & $-0,25$ \\
\hline 3 & $-1,31$ & 55,22 & 53,68 & 1,08 & 0,46 & & $-0,46$ & $-1,08$ \\
\hline 4 & 3,47 & 37,24 & 40,67 & $-0,86$ & $-2,58$ & & 2,58 & 0,85 \\
\hline 5 & $-0,16$ & 25,20 & 24,76 & 0,43 & 0,00 & & 0,00 & $-0,43$ \\
\hline 6 & 0,17 & 21,69 & 21,77 & $-0,12$ & 0,04 & & $-0,04$ & 0,12 \\
\hline 7 & 0,20 & 24,40 & 24,08 & 0,04 & 0,28 & & $-0,28$ & $-0,04$ \\
\hline 8 & $-0,38$ & 156,24 & 155,83 & 0,68 & $-0,27$ & & 0,27 & $-0,68$ \\
\hline 9 & $-0,50$ & 80,16 & 79,17 & 0,57 & 0,42 & & $-0,42$ & $-0,57$ \\
\hline 10 & 0,08 & 28,39 & 28,59 & 0,01 & $-0,21$ & & 0,21 & $-0,01$ \\
\hline 11 & 14,84 & 23,32 & 24,49 & $-0,14$ & $-1,04$ & & 1,04 & 0,13 \\
\hline & & & & 4,33 & 6,33 & $\mathbf{T A D}^{\mathrm{c}}$ & 6,32 & 4,33 \\
\hline
\end{tabular}

a) correcting factor: $\mathrm{CF}_{\mathrm{i}}=\mathrm{m}_{\mathrm{i}}^{\text {epimers }}-\mathrm{m}_{\mathrm{i}}{ }^{\mathrm{DB}}$, where $\mathrm{m}_{\mathrm{i}}$ 's are mean chemical shift values

b) adjusted chemical shift values, obtained $c . s_{{ }_{\mathrm{i}}}=\mathrm{c} . \mathrm{s}_{._{\mathrm{i}}}-\mathrm{CF}$

c) $\mathrm{TAD}=$ Total Absolute Deviation: sum of absolute values of all c.s. differences $=\Sigma_{\mathrm{i}}\left|\Delta \delta_{\mathrm{i}}\right|$

Figure S2: Histograms of modified UDB analysis for 2'-tertbutyl taxanes major vs minor

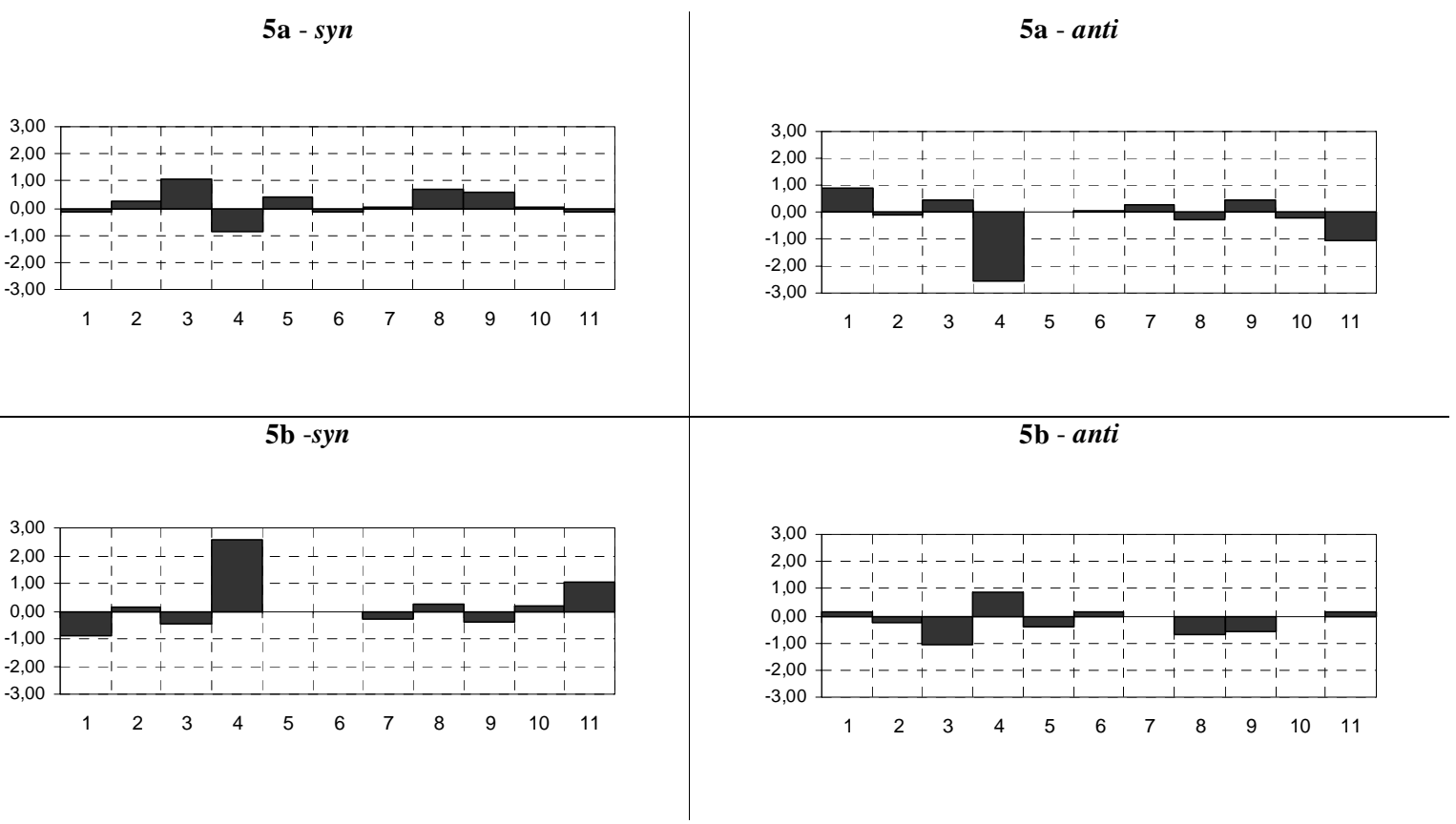


D) Comparison of the minor conformers of epimer 1 of 2'-tertbutyl taxanes and the major confermer of its diastereomer with epimeric 2'-methyltaxanes

Table S24: Side chain ${ }^{13} \mathrm{C}$ NMR c.s. values of the epimeric 2 -methyl taxanes and the compounds under investigation

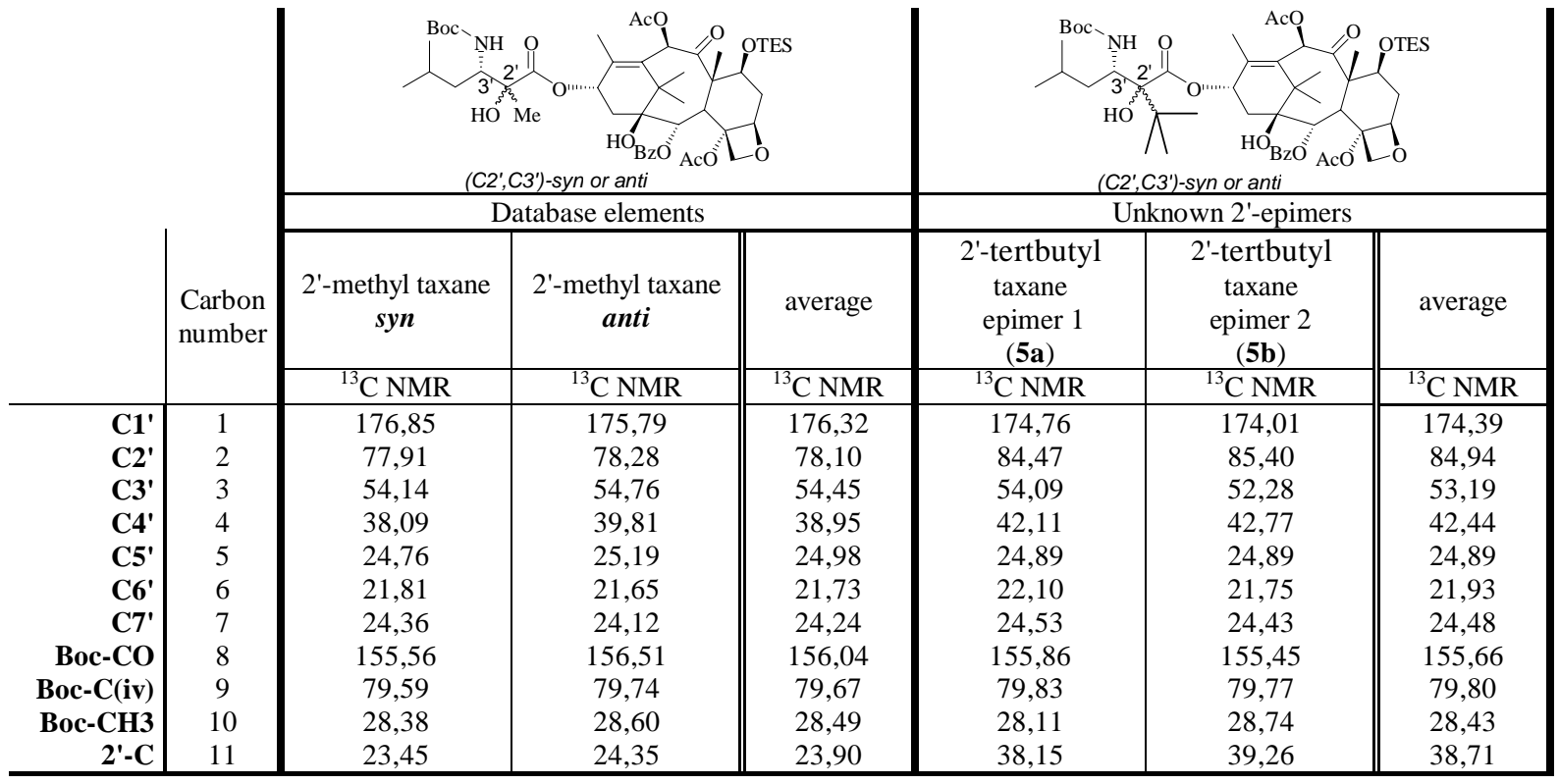

\section{Original UBD analysis}

Table S25: Original UDB analysis performed on title compound

\begin{tabular}{|c|c|c|c|c|c|c|c|c|c|c|}
\hline \multirow{4}{*}{$\begin{array}{l}\text { Carbon } \\
\text { number }\end{array}$} & \multicolumn{2}{|c|}{ Calculated c.s. ${ }^{\mathrm{a}}$} & \multirow{3}{*}{$\begin{array}{l}\text { Correcting } \\
\text { factor }^{\mathrm{b}}\end{array}$} & \multicolumn{2}{|c|}{ Adjusted c.s. ${ }^{c}$} & \multicolumn{5}{|c|}{ Chemical shift difference } \\
\hline & \multirow[b]{2}{*}{$\begin{array}{l}2 \text { 'methyl } \\
\text { taxane }\end{array}$} & \multirow[b]{2}{*}{$\begin{array}{l}2^{\prime}- \\
\text { tertbutyl } \\
\text { taxane }\end{array}$} & & \multirow{2}{*}{$\begin{array}{c}2^{\prime}- \\
\text { tertbutyl } \\
\text { taxane } \\
\text { epimer } 1 \\
\text { (5a) }\end{array}$} & \multirow{2}{*}{$\begin{array}{c}2^{\prime}- \\
\text { tertbutyl } \\
\text { taxane } \\
\text { epimer } 2 \\
(\mathbf{5 b})\end{array}$} & \multicolumn{5}{|c|}{ (2'-methyl taxane) - (adjusted 2'-tertbutyl taxane) } \\
\hline & & & & & & syn $-\mathbf{5 a}$ & anti - 5a & & syn $-\mathbf{5 b}$ & anti - $\mathbf{5 b}$ \\
\hline & $\begin{array}{c}{ }^{13} \mathrm{C} \\
\mathrm{NMR}\end{array}$ & $\begin{array}{c}{ }^{13} \mathrm{C} \\
\mathrm{NMR}\end{array}$ & ${ }^{13} \mathrm{C}$ NMR & ${ }^{13} \mathrm{C}$ NMR & ${ }^{13} \mathrm{C}$ NMR & ${ }^{13} \mathrm{C}$ NMR & ${ }^{13} \mathrm{C}$ NMR & & ${ }^{13} \mathrm{C} \mathrm{NMR}$ & ${ }^{13} \mathrm{C}$ NMR \\
\hline 1 & 174,50 & 176,00 & 1,50 & 173,26 & 172,51 & $-3,59$ & $-2,53$ & & $-4,34$ & $-3,28$ \\
\hline 2 & 87,90 & 105,10 & 17,20 & 67,27 & 68,20 & $-10,64$ & $-11,01$ & & $-9,71$ & $-10,08$ \\
\hline 3 & 55,30 & 47,80 & $-7,50$ & 61,59 & 59,78 & 7,45 & 6,83 & & 5,64 & 5,02 \\
\hline 4 & 36,10 & 37,00 & 0,90 & 41,21 & 41,87 & 3,12 & 1,40 & & 3,78 & 2,06 \\
\hline 5 & 23,70 & 23,70 & 0,00 & 24,89 & 24,89 & 0,13 & $-0,30$ & & 0,13 & $-0,30$ \\
\hline 6 & 22,30 & 22,30 & 0,00 & 22,10 & 21,75 & 0,29 & 0,45 & & $-0,06$ & 0,10 \\
\hline 7 & 22,30 & 22,30 & 0,00 & 24,53 & 24,43 & 0,17 & 0,41 & & 0,07 & 0,31 \\
\hline 8 & 157,20 & 157,20 & 0,00 & 155,86 & 155,45 & 0,30 & $-0,65$ & & $-0,11$ & $-1,06$ \\
\hline 9 & 70,60 & 70,60 & 0,00 & 79,83 & 79,77 & 0,24 & 0,09 & & 0,18 & 0,03 \\
\hline 10 & 28,70 & 28,70 & 0,00 & 28,11 & 28,74 & $-0,27$ & $-0,49$ & & 0,36 & 0,14 \\
\hline 11 & 17,90 & 31,60 & 13,70 & 24,45 & 25,56 & 1,00 & 0,10 & & 2,11 & 1,21 \\
\hline & & & & & & 27,20 & 24,26 & $\begin{array}{c}\mathbf{T} \\
\mathbf{A} \\
\mathbf{D}^{\mathrm{d}} \\
\end{array}$ & 26,49 & 23,59 \\
\hline
\end{tabular}

a) CS ChemNMR Pro version 1.0, Renate Buergin Schaller, Development Centre, Bergstr. 114, Zurich, Switzerland, installed in CS ChemDraw Pro version 7.0, was used.

b) Correcting factor $=($ database elements c.s. calculated value $)$ (unknown 2 '-epimers c.s. calculated value $)$

c) adjusted c.s. value $=($ unknown 2 '-epimer c.s. measured value $)+($ correcting factor $)$

d) $\mathrm{TAD}=$ Total Absolute Deviation: sum of absolute values of all c.s. differences $=\Sigma_{\mathrm{i}}\left|\Delta \delta_{\mathrm{i}}\right|$ 


\section{Modified UDB approach}

Table S26: Modified UDB analysis performed on title compound.

\begin{tabular}{|c|c|c|c|c|c|c|c|c|}
\hline \multirow{4}{*}{$\begin{array}{l}\text { Carbon } \\
\text { number }\end{array}$} & \multirow[b]{3}{*}{$\begin{array}{l}\text { Correcting } \\
\text { factor }^{\mathrm{a}}\end{array}$} & \multicolumn{2}{|c|}{ Adjusted c.s. values ${ }^{b}$} & \multicolumn{5}{|c|}{ Chemical shift difference $\Delta \delta_{i}$} \\
\hline & & \multirow{2}{*}{$\begin{array}{c}2^{\prime}- \\
\text { tertbutyl } \\
\text { taxane } \\
\text { epimer } 1 \\
\text { (5a) }\end{array}$} & \multirow{2}{*}{$\begin{array}{c}2^{\prime}- \\
\text { tertbutyl } \\
\text { taxane } \\
\text { epimer } 2 \\
(\mathbf{5 b})\end{array}$} & \multicolumn{5}{|c|}{ (2'-tertbutyl taxane) - (2'-methyl taxane) } \\
\hline & & & & $5 \mathbf{a}-s y n$ & $5 \mathbf{a}-$ anti & & $5 \mathbf{b}-s y n$ & $5 \mathbf{b}-$ anti \\
\hline & ${ }^{13} \mathrm{C}$ NMR & ${ }^{13} \mathrm{C}$ NMR & ${ }^{13} \mathrm{C}$ NMR & ${ }^{13} \mathrm{C}$ NMR & ${ }^{13} \mathrm{C} \mathrm{NMR}$ & & ${ }^{13} \mathrm{C} \mathrm{NMR}$ & ${ }^{13} \mathrm{C} \mathrm{NMR}$ \\
\hline 1 & $-1,94$ & 176,70 & 175,95 & $-0,16$ & 0,91 & & $-0,91$ & 0,16 \\
\hline 2 & 6,84 & 77,63 & 78,56 & $-0,28$ & $-0,65$ & & 0,65 & 0,28 \\
\hline 3 & $-1,27$ & 55,36 & 53,55 & 1,22 & 0,60 & & $-0,59$ & $-1,22$ \\
\hline 4 & 3,49 & 38,62 & 39,28 & 0,53 & $-1,19$ & & 1,19 & $-0,53$ \\
\hline 5 & $-0,09$ & 24,98 & 24,98 & 0,22 & $-0,22$ & & 0,22 & $-0,22$ \\
\hline 6 & 0,20 & 21,91 & 21,56 & 0,09 & 0,25 & & $-0,26$ & $-0,10$ \\
\hline 7 & 0,24 & 24,29 & 24,19 & $-0,07$ & 0,17 & & $-0,17$ & 0,07 \\
\hline 8 & $-0,38$ & 156,24 & 155,83 & 0,68 & $-0,27$ & & 0,27 & $-0,68$ \\
\hline 9 & 0,14 & 79,70 & 79,64 & 0,10 & $-0,05$ & & 0,04 & $-0,11$ \\
\hline 10 & $-0,07$ & 28,18 & 28,81 & $-0,20$ & $-0,42$ & & 0,43 & 0,21 \\
\hline 11 & 14,81 & 23,35 & 24,46 & $-0,11$ & $-1,01$ & & 1,01 & 0,10 \\
\hline & & & & 3,65 & 5,72 & TAD $^{c}$ & 5,72 & 3,66 \\
\hline
\end{tabular}

a) correcting factor: $\mathrm{CF}_{i}=\mathrm{m}_{\mathrm{i}}^{\text {epimers }}-\mathrm{m}_{\mathrm{i}}^{\mathrm{DB}}$, where $\mathrm{m}_{\mathrm{i}}$ 's are mean chemical shift values

b) adjusted chemical shift values, obtained $c . s_{\cdot}{ }_{\mathrm{i}}=\mathrm{c} . \mathrm{s}_{\mathrm{i}_{\mathrm{i}}}-\mathrm{CF}_{\mathrm{i}}$

c) $\mathrm{TAD}=$ Total Absolute Deviation: sum of absolute values of all c.s. differences $=\Sigma_{\mathrm{i}}\left|\Delta \delta_{\mathrm{i}}\right|$

Figure S3: Histograms of modified UDB analysis for 2'-tertbutyl taxanes minor vs major

$5 \mathbf{a}-\operatorname{syn}$

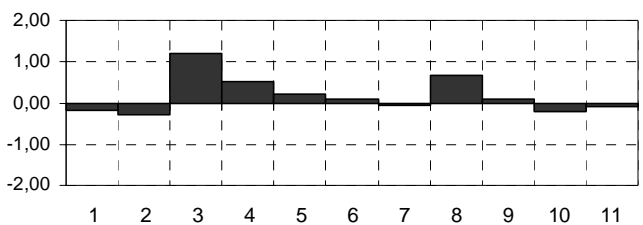

5a - anti

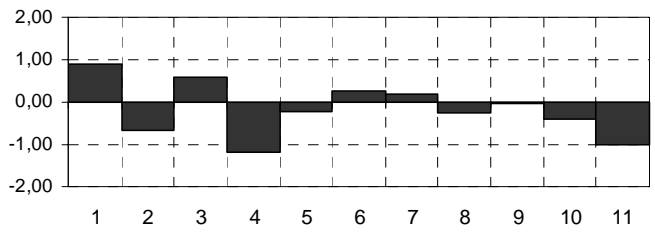

$5 \mathbf{b}-\mathbf{a n t i}$

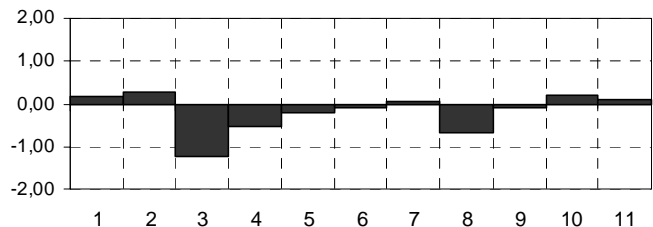




\section{2'-Phenyltaxanes:}

Table S27: Side chain ${ }^{13} \mathrm{C}$ NMR c.s. values of the epimeric 2 -methyl taxanes and the compounds under investigation

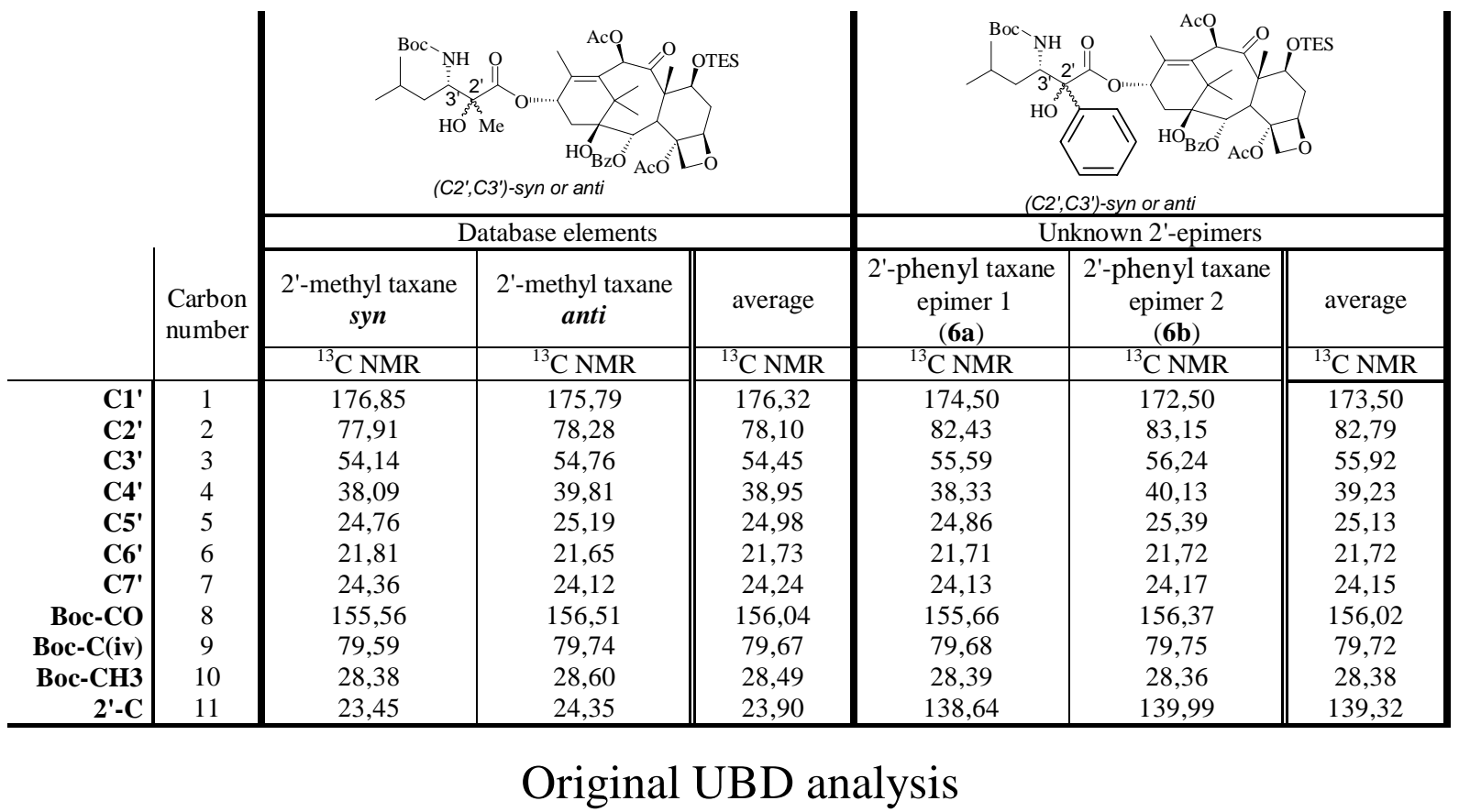

Table S28: Original UDB analysis performed on title compound

\begin{tabular}{|c|c|c|c|c|c|c|c|c|c|c|}
\hline \multirow{4}{*}{$\begin{array}{l}\text { Carbon } \\
\text { number }\end{array}$} & \multicolumn{2}{|c|}{ Calculated c.s. $^{\text {a }}$} & \multirow{3}{*}{$\begin{array}{l}\text { Correcting } \\
\text { factor }^{b}\end{array}$} & \multicolumn{2}{|c|}{ Adjusted c.s. ${ }^{c}$} & \multicolumn{5}{|c|}{ Chemical shift difference } \\
\hline & \multirow[b]{2}{*}{$\begin{array}{l}\text { 2'methyl } \\
\text { taxane }\end{array}$} & \multirow{2}{*}{$\begin{array}{l}2^{2}- \\
\text { phenyl } \\
\text { taxane }\end{array}$} & & \multirow{2}{*}{$\begin{array}{l}\text { 2'-phenyl } \\
\text { taxane } \\
\text { epimer } 1 \\
(\mathbf{6 a})\end{array}$} & \multirow{2}{*}{$\begin{array}{l}\text { 2'-phenyl } \\
\text { taxane } \\
\text { epimer } 2 \\
(\mathbf{6 b})\end{array}$} & \multicolumn{5}{|c|}{ (2'-methyl taxane) - (adjusted 2'-phenyl taxane) } \\
\hline & & & & & & syn-6a & anti- $\mathbf{6 a}$ & & $s y n-6 b$ & $a n t i-6 b$ \\
\hline & $\begin{array}{c}{ }^{13} \mathrm{C} \\
\mathrm{NMR}\end{array}$ & $\begin{array}{c}{ }^{13} \mathrm{C} \\
\mathrm{NMR}\end{array}$ & ${ }^{13} \mathrm{C}$ NMR & ${ }^{13} \mathrm{C}$ NMR & ${ }^{13} \mathrm{C}$ NMR & ${ }^{13} \mathrm{C}$ NMR & ${ }^{13} \mathrm{C}$ NMR & & ${ }^{13} \mathrm{C}$ NMR & ${ }^{13} \mathrm{C}$ NMR \\
\hline 1 & 174,50 & 173,00 & 1,50 & 176,00 & 174,00 & 0,85 & $-0,21$ & & 2,85 & 1,79 \\
\hline 2 & 87,90 & 94,60 & $-6,70$ & 75,73 & 76,45 & 2,18 & 2,55 & & 1,46 & 1,83 \\
\hline 3 & 55,30 & 55,20 & 0,10 & 55,69 & 56,34 & $-1,55$ & $-0,93$ & & $-2,20$ & $-1,58$ \\
\hline 4 & 36,10 & 36,00 & 0,10 & 38,43 & 40,23 & $-0,34$ & 1,38 & & $-2,14$ & $-0,42$ \\
\hline 5 & 23,70 & 23,70 & 0,00 & 24,86 & 25,39 & $-0,10$ & 0,33 & & $-0,63$ & $-0,20$ \\
\hline 6 & 22,30 & 22,30 & 0,00 & 21,71 & 21,72 & 0,10 & $-0,06$ & & 0,09 & $-0,07$ \\
\hline 7 & 22,30 & 22,30 & 0,00 & 24,13 & 24,17 & 0,23 & $-0,01$ & & 0,19 & $-0,05$ \\
\hline 8 & 157,20 & 157,20 & 0,00 & 155,66 & 156,37 & $-0,10$ & 0,85 & & $-0,81$ & 0,14 \\
\hline 9 & 70,60 & 70,60 & 0,00 & 79,68 & 79,75 & $-0,09$ & 0,06 & & $-0,16$ & $-0,01$ \\
\hline 10 & 28,70 & 28,70 & 0,00 & 28,39 & 28,36 & $-0,01$ & 0,21 & & 0,02 & 0,24 \\
\hline 11 & 17,90 & 139,40 & $-121,50$ & 17,14 & 18,49 & 6,31 & 7,21 & & 4,96 & 5,86 \\
\hline & & & & & & 11,86 & 13,80 & $\begin{array}{c}\mathbf{T} \\
\mathbf{A} \\
\mathbf{D}^{\mathrm{d}}\end{array}$ & 15,51 & 12,19 \\
\hline
\end{tabular}

a) CS ChemNMR Pro version 1.0, Renate Buergin Schaller, Development Centre, Bergstr. 114, Zurich, Switzerland, installed in CS ChemDraw Pro version 7.0, was used.

b) Correcting factor $=($ database elements c.s. calculated value $)$ (unknown 2 '-epimers c.s. calculated value $)$

c) adjusted c.s. value $=($ unknown 2 '-epimer c.s. measured value $)+($ correcting factor $)$

d) $\mathrm{TAD}=$ Total Absolute Deviation: sum of absolute values of all c.s. differences $=\Sigma_{\mathrm{i}}\left|\Delta \delta_{\mathrm{i}}\right|$ 


\section{Modified UDB approach}

Table S29: $\quad$ Modified UDB analysis performed on title compound.

\begin{tabular}{|c|c|c|c|c|c|c|c|c|}
\hline \multirow{4}{*}{$\begin{array}{l}\text { Carbon } \\
\text { number }\end{array}$} & \multirow{3}{*}{$\begin{array}{l}\text { Correcting } \\
\text { factor }^{\mathrm{a}}\end{array}$} & \multicolumn{2}{|c|}{ Adjusted c.s. values ${ }^{b}$} & \multirow{2}{*}{\multicolumn{5}{|c|}{$\frac{\text { Chemical shift difference } \Delta \delta_{i}}{\text { (2'-phenyl taxane) }-\left(2^{\prime} \text {-methyl taxane }\right)}$}} \\
\hline & & \multirow{2}{*}{$\begin{array}{c}\text { 2'-phenyl } \\
\text { taxane } \\
\text { epimer } 1 \\
(\mathbf{6 a})\end{array}$} & \multirow{2}{*}{$\begin{array}{c}\text { 2'-phenyl } \\
\text { taxane } \\
\text { epimer } 2 \\
(\mathbf{6 b})\end{array}$} & & & & & \\
\hline & & & & $6 a-s y n$ & $6 \mathbf{a}-a n t i$ & & $6 \mathrm{~b}-s y n$ & $6 b-a n t i$ \\
\hline & ${ }^{13} \mathrm{C}$ NMR & ${ }^{13} \mathrm{C}$ NMR & ${ }^{13} \mathrm{C}$ NMR & ${ }^{13} \mathrm{C}$ NMR & ${ }^{13} \mathrm{C}$ NMR & & ${ }^{13} \mathrm{C}$ NMR & ${ }^{13} \mathrm{C} \mathrm{NMR}$ \\
\hline 1 & $-2,82$ & 177,32 & 175,32 & 0,47 & 1,53 & & $-1,53$ & $-0,47$ \\
\hline 2 & 4,70 & 77,74 & 78,46 & $-0,17$ & $-0,55$ & & 0,55 & 0,17 \\
\hline 3 & 1,47 & 54,13 & 54,78 & $-0,02$ & $-0,63$ & & 0,63 & 0,02 \\
\hline 4 & 0,28 & 38,05 & 39,85 & $-0,04$ & $-1,76$ & & 1,76 & 0,04 \\
\hline 5 & 0,15 & 24,71 & 25,24 & $-0,05$ & $-0,48$ & & 0,48 & 0,05 \\
\hline 6 & $-0,01$ & 21,73 & 21,74 & $-0,09$ & 0,07 & & $-0,08$ & 0,08 \\
\hline 7 & $-0,09$ & 24,22 & 24,26 & $-0,14$ & 0,10 & & $-0,10$ & 0,14 \\
\hline 8 & $-0,02$ & 155,68 & 156,39 & 0,12 & $-0,83$ & & 0,83 & $-0,12$ \\
\hline 9 & 0,05 & 79,63 & 79,70 & 0,04 & $-0,11$ & & 0,11 & $-0,04$ \\
\hline 10 & $-0,12$ & 28,51 & 28,48 & 0,13 & $-0,09$ & & 0,10 & $-0,13$ \\
\hline 11 & 115,42 & 23,23 & 24,58 & $-0,23$ & $-1,13$ & & 1,13 & 0,23 \\
\hline & & & & 1,49 & 7,29 & $\mathbf{T A D}^{\mathrm{c}}$ & 7,29 & 1,49 \\
\hline
\end{tabular}

a) correcting factor: $\mathrm{CF}_{\mathrm{i}}=\mathrm{m}_{\mathrm{i}}^{\text {epimers }}-\mathrm{m}_{\mathrm{i}}^{\mathrm{DB}}$, where $\mathrm{m}_{\mathrm{i}}$ 's are mean chemical shift values

b) adjusted chemical shift values, obtained $C . s_{\cdot \mathrm{i}}=\mathrm{C} . \mathrm{S}_{_{\textrm{i}}}-\mathrm{CF}_{\mathrm{i}}$

c) $\mathrm{TAD}=$ Total Absolute Deviation: sum of absolute values of all c.s. differences $=\Sigma_{\mathrm{i}}\left|\Delta \delta_{\mathrm{i}}\right|$

Epimer $1(\mathbf{6 a}):$ Syn

Epimer 2 (6b): $\quad$ Anti 


\section{$\underline{\text { 2'-Vinyltaxanes: }}$}

Table S30: Side chain ${ }^{13} \mathrm{C}$ NMR c.s. values of the epimeric 2'-methyl taxanes and the compounds under investigation

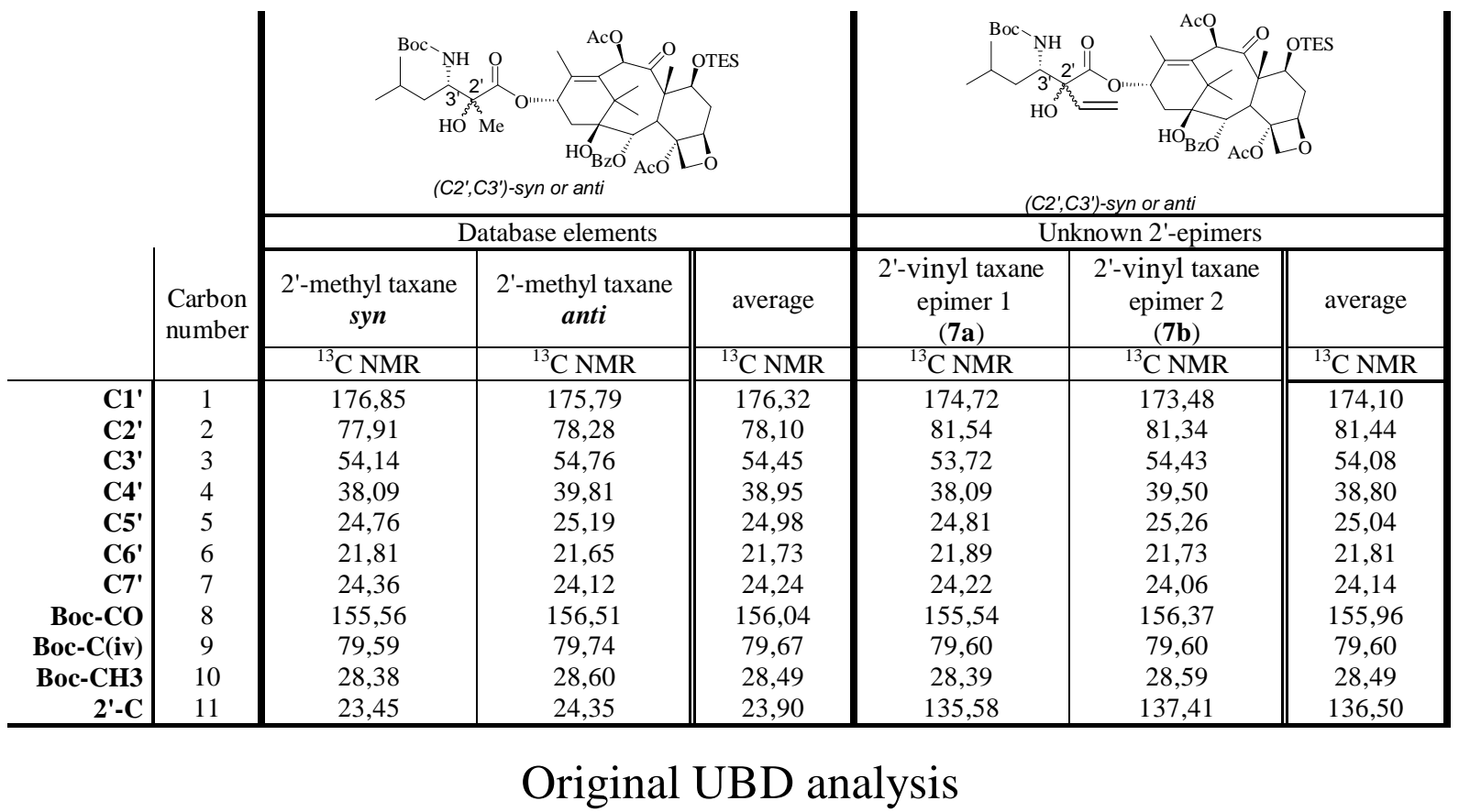

Table S31: Original UDB analysis performed on title compound

\begin{tabular}{|c|c|c|c|c|c|c|c|c|c|c|}
\hline \multirow{4}{*}{$\begin{array}{l}\text { Carbon } \\
\text { number }\end{array}$} & \multicolumn{2}{|c|}{ Calculated c.s. $^{\text {a }}$} & \multirow{3}{*}{$\begin{array}{l}\text { Correcting } \\
\text { factor }^{b}\end{array}$} & \multicolumn{2}{|c|}{ Adjusted c.s. ${ }^{c}$} & \multicolumn{5}{|c|}{ Chemical shift difference } \\
\hline & \multirow[b]{2}{*}{$\begin{array}{l}\text { 2'methyl } \\
\text { taxane }\end{array}$} & \multirow[b]{2}{*}{$\begin{array}{l}\text { 2'-vinyl } \\
\text { taxane }\end{array}$} & & \multirow{2}{*}{$\begin{array}{l}\text { 2'-vinyl } \\
\text { taxane } \\
\text { epimer } 1 \\
(\mathbf{7 a})\end{array}$} & \multirow{2}{*}{$\begin{array}{l}\text { 2'-vinyl } \\
\text { taxane } \\
\text { epimer } 2 \\
(\mathbf{7 b})\end{array}$} & \multicolumn{5}{|c|}{ (2'-methyl taxane $)$ - (adjusted 2'-vinyl taxane) } \\
\hline & & & & & & $s y n-7 a$ & anti $-7 \mathbf{a}$ & & $s y n-7 b$ & anti $-7 \mathrm{~b}$ \\
\hline & $\begin{array}{c}{ }^{13} \mathrm{C} \\
\mathrm{NMR}\end{array}$ & $\begin{array}{c}{ }^{13} \mathrm{C} \\
\mathrm{NMR}\end{array}$ & ${ }^{13} \mathrm{C}$ NMR & ${ }^{13} \mathrm{C}$ NMR & ${ }^{13} \mathrm{C}$ NMR & ${ }^{13} \mathrm{C}$ NMR & ${ }^{13} \mathrm{C}$ NMR & & ${ }^{13} \mathrm{C}$ NMR & ${ }^{13} \mathrm{C}$ NMR \\
\hline 1 & 174,50 & 172,00 & 2,50 & 177,22 & 175,98 & $-0,37$ & $-1,43$ & & 0,87 & $-0,19$ \\
\hline 2 & 87,90 & 91,80 & $-3,90$ & 77,64 & 77,44 & 0,27 & 0,64 & & 0,47 & 0,84 \\
\hline 3 & 55,30 & 52,80 & 2,50 & 56,22 & 56,93 & $-2,08$ & $-1,46$ & & $-2,79$ & $-2,17$ \\
\hline 4 & 36,10 & 36,50 & $-0,40$ & 37,69 & 39,10 & 0,40 & 2,12 & & $-1,01$ & 0,71 \\
\hline 5 & 23,70 & 23,80 & $-0,10$ & 24,71 & 25,16 & 0,05 & 0,48 & & $-0,40$ & 0,03 \\
\hline 6 & 22,30 & 22,30 & 0,00 & 21,89 & 21,73 & $-0,08$ & $-0,24$ & & 0,08 & $-0,08$ \\
\hline 7 & 22,30 & 22,30 & 0,00 & 24,22 & 24,06 & 0,14 & $-0,10$ & & 0,30 & 0,06 \\
\hline 8 & 157,20 & 157,20 & 0,00 & 155,54 & 156,37 & 0,02 & 0,97 & & $-0,81$ & 0,14 \\
\hline 9 & 70,60 & 70,60 & 0,00 & 79,60 & 79,60 & $-0,01$ & 0,14 & & $-0,01$ & 0,14 \\
\hline 10 & 28,70 & 28,70 & 0,00 & 28,39 & 28,59 & $-0,01$ & 0,21 & & $-0,21$ & 0,01 \\
\hline 11 & 17,90 & 140,60 & $-122,70$ & 12,88 & 14,71 & 10,57 & 11,47 & & 8,74 & 9,64 \\
\hline & & & & & & 14,00 & 19,26 & $\begin{array}{c}\mathbf{T} \\
\mathbf{A} \\
\mathbf{D}^{\mathrm{d}}\end{array}$ & 15,69 & 14,01 \\
\hline
\end{tabular}

a) CS ChemNMR Pro version 1.0, Renate Buergin Schaller, Development Centre, Bergstr. 114, Zurich, Switzerland, installed in CS ChemDraw Pro version 7.0, was used.

b) Correcting factor $=($ database elements c.s. calculated value $)$ ( unknown 2 '-epimers c.s. calculated value $)$

c) adjusted c.s. value $=($ unknown 2 '-epimer c.s. measured value $)+($ correcting factor $)$

d) $\mathrm{TAD}=$ Total Absolute Deviation: sum of absolute values of all c.s. differences $=\Sigma_{\mathrm{i}}\left|\Delta \delta_{\mathrm{i}}\right|$ 


\section{Modified UDB approach}

Table S32: Modified UDB analysis performed on title compound.

\begin{tabular}{|c|c|c|c|c|c|c|c|c|}
\hline \multirow{4}{*}{$\begin{array}{l}\text { Carbon } \\
\text { number }\end{array}$} & \multirow{3}{*}{$\begin{array}{l}\text { Correcting } \\
\text { factor }^{\mathrm{a}}\end{array}$} & \multicolumn{2}{|c|}{ Adjusted c.s. values ${ }^{\mathrm{b}}$} & \multirow{2}{*}{\multicolumn{5}{|c|}{$\frac{\text { Chemical shift difference } \Delta \delta_{\mathrm{i}}}{\text { (2'-vinyl taxane) }-\left(2^{\prime} \text {-methyl taxane }\right)}$}} \\
\hline & & \multirow{2}{*}{$\begin{array}{l}\text { 2'-vinyl } \\
\text { taxane } \\
\text { epimer } 1 \\
\text { (7a) }\end{array}$} & \multirow{2}{*}{$\begin{array}{c}\text { 2'-vinyl } \\
\text { taxane } \\
\text { epimer } 2 \\
(7 \mathbf{b})\end{array}$} & & & & & \\
\hline & & & & $7 \mathbf{a}-s y n$ & $7 \mathbf{a}-$ anti & & $7 b-s y n$ & $7 b-a n t i$ \\
\hline & ${ }^{13} \mathrm{C}$ NMR & ${ }^{13} \mathrm{C} \mathrm{NMR}$ & ${ }^{13} \mathrm{C} \mathrm{NMR}$ & ${ }^{13} \mathrm{C}$ NMR & ${ }^{13} \mathrm{C} \mathrm{NMR}$ & & ${ }^{13} \mathrm{C} \mathrm{NMR}$ & ${ }^{13} \mathrm{C}$ NMR \\
\hline 1 & $-2,22$ & 176,94 & 175,70 & 0,09 & 1,15 & & $-1,15$ & $-0,09$ \\
\hline 2 & 3,35 & 78,20 & 78,00 & 0,29 & $-0,08$ & & 0,09 & $-0,28$ \\
\hline 3 & $-0,38$ & 54,10 & 54,81 & $-0,05$ & $-0,66$ & & 0,66 & 0,05 \\
\hline 4 & $-0,16$ & 38,25 & 39,66 & 0,16 & $-1,57$ & & 1,57 & $-0,16$ \\
\hline 5 & 0,06 & 24,75 & 25,20 & $-0,01$ & $-0,44$ & & 0,44 & 0,01 \\
\hline 6 & 0,08 & 21,81 & 21,65 & 0,00 & 0,16 & & $-0,16$ & 0,00 \\
\hline 7 & $-0,10$ & 24,32 & 24,16 & $-0,04$ & 0,20 & & $-0,20$ & 0,04 \\
\hline 8 & $-0,08$ & 155,62 & 156,45 & 0,06 & $-0,89$ & & 0,89 & $-0,06$ \\
\hline 9 & $-0,06$ & 79,67 & 79,67 & 0,07 & $-0,08$ & & 0,07 & $-0,08$ \\
\hline 10 & 0,00 & 28,39 & 28,59 & 0,01 & $-0,21$ & & 0,21 & $-0,01$ \\
\hline 11 & 112,60 & 22,99 & 24,82 & $-0,46$ & $-1,36$ & & 1,37 & 0,46 \\
\hline & & & & 1,24 & 6,80 & $\mathbf{T A D}^{\mathrm{c}}$ & 6,81 & 1,23 \\
\hline
\end{tabular}

a) correcting factor: $\mathrm{CF}_{\mathrm{i}}=\mathrm{m}_{\mathrm{i}}^{\text {epimers }}-\mathrm{m}_{\mathrm{i}}^{\mathrm{DB}}$, where $\mathrm{m}_{\mathrm{i}}$ 's are mean chemical shift values

b) adjusted chemical shift values, obtained $c . s_{\cdot{ }_{\mathrm{i}}}=\mathrm{C} . \mathrm{S}_{_{\textrm{i}}}-\mathrm{CF}_{\mathrm{i}}$

c) $\mathrm{TAD}=$ Total Absolute Deviation: sum of absolute values of all c.s. differences $=\Sigma_{\mathrm{i}}\left|\Delta \delta_{\mathrm{i}}\right|$

Epimer 1 (7a): $\quad$ Syn

Epimer 2 (7b): $\quad$ Anti 